OPEN ACCESS

Edited by:

Chi-Ming Wong,

Hong Kong Polytechnic University,

Hong Kong

Reviewed by:

Chi Ping Chan

The University of Hong Kong,

Hong Kong

Petar Ozretić,

Rudjer Boskovic Institute, Croatia

*Correspondence:

Ninghong Song

songninghong_urol@163.com

Xianghu Meng

xhmeng888@163.com

xhmeng@njmu.edu.cn

${ }^{\dagger}$ These authors have contributed

equally to this work

Specialty section:

This article was submitted to

$R N A$,

a section of the journa

Frontiers in Genetics

Received: 10 October 2020

Accepted: 08 February 2021

Published: 11 March 2021

Citation:

Yao L, Cong R, Ji C, Zhou X,

Luan J, Meng X and Song N (2021)

RNA-Binding Proteins Play an

Important Role in the Prognosis

of Patients With Testicular Germ Cell

Tumor. Front. Genet. 12:610291.

doi: 10.3389/fgene.2021.610291

\section{RNA-Binding Proteins Play an Important Role in the Prognosis of Patients With Testicular Germ Cell Tumor}

\author{
Liangyu Yao ${ }^{1 t}$, Rong Cong ${ }^{1 t}$, Chengjian $\mathrm{Ji}^{1+}$, Xiang Zhou ${ }^{1}$, Jiaochen Luan ${ }^{1}$, \\ Xianghu Meng ${ }^{1 *}$ and Ninghong Song ${ }^{1,2 *}$
}

1 Department of Urology, The First Affiliated Hospital of Nanjing Medical University, Nanjing, China, ${ }^{2}$ Department of Urology, The Affiliated Kizilsu Kirghiz Autonomous Prefecture People's Hospital of Nanjing Medical University, Artux, China

Testicular germ cell tumors (TGCTs) are common urological neoplasms in young adult males. The outcome of TGCT depends on pathologic type and tumor stage. RNAbinding proteins (RBPs) influence numerous cancers via post-transcriptional regulation. The prognostic importance of RBPs in TGCT has not been fully investigated. In this study, we set up a prognostic risk model of TGCT using six significantly differentially expressed RBPs, namely, TRMT61A, POLR2J, DIS3L2, IFIH1, IGHMBP2, and NPM2. The expression profiles were downloaded from The Cancer Genome Atlas (TCGA) and Genotype-Tissue Expression datasets. We observed by performing least absolute shrinkage and selection operator (LASSO) regression analyses that in the training cohort, the expression of six RBPs was correlated with disease-free survival in patients with TGCT. We assessed the specificity and sensitivity of 1-, 3-, 5-, and 10-year survival status prediction using receiver operating characteristic curve analysis and successfully validated using the test cohorts, the entire TCGA cohort, and Gene Expression Omnibus (GEO) datasets. Gene Ontology, Kyoto Encyclopedia of Genes and Genomes, and gene set enrichment analyses were carried out to seek the possible signaling pathways related with risk score. We also examined the association between the model based on six RBPs and different clinical characteristics. A nomogram was established for TGCT recurrence prediction. Consensus clustering analysis was carried out to identify the clusters of TGCT with different clinical outcomes. Ultimately, external validations of the six-gene risk score were performed by using the GSE3218 and GSE10783 datasets downloaded from the GEO database. In general, our study constructed a prognostic model based on six RBPs, which could serve as independent risk factor in TGCT, especially in seminoma, and might have brilliant clinical application value.

Keywords: testicular germ cell tumors, RNA-binding proteins, risk score, prognosis, nomogram

\section{INTRODUCTION}

Testicular germ cell tumors (TGCTs) are the most common malignant neoplasms in young adult males between 20 and 40 years old despite being regarded as rare tumor types that account for only $1 \%$ of solid neoplasms in men (Tsili et al., 2019). TGCT has two main subtypes: seminoma and non-seminoma. The outcome depends on its pathologic type and tumor stage (Zhang et al., 2018). 
Although TGCT mortality is lower than $10 \%$ and the cure rate has reached $95 \%$ because of the application of cisplatin-based chemotherapy (Diamantopoulos and Kortsaris, 2010; Cheng et al., 2018), 10-15\% of the patients are refractory to firstline chemotherapy and TGCT has poor outcome (Oechsle et al., 2011). Serum tumor markers, such as alpha fetoprotein (AFP), human chorionic gonadotropin (hCG), and lactose dehydrogenase (LDH), are widely applied clinically and effective in the diagnosis, staging, risk stratification, treatment, and evaluation of patients' response to chemotherapy in TGCT (Marshall et al., 2019). Several studies also attempted to look for biomarkers related with prognosis of TGCT. MGMT and CALCA promoter methylation were found in connection with poor outcome in patients with TGCT (Martinelli et al., 2017). $\beta$-catenin was linked to suppressed immune infiltration and poor clinical features in TGCT (Chovanec et al., 2018). However, a risk model for TGCT prognosis prediction has not been designed so far.

RNA-binding proteins (RBPs) are identified as conserved proteins in eukaryotes that play critical roles in co-transcriptional and post-transcriptional gene regulation, particularly RNA maturation, turnover, localization, and translation (Glisovic et al., 2008). The dysregulation of RBPs in diverse cancers influences the expression and function of tumor-related proteins through mechanisms, such as post-transcriptional regulations, RBPRNA networks, and results in cancer development (Pereira et al., 2017). The musashi RNA binding protein 2 stabilizes androgen receptor mRNA by targeting its $3^{\prime}$-untranslated region and drives prostate cancer progression (Zhao et al., 2020). Similarly, KHDRBS1 functions as a post-transcriptional regulator by binding to its target mRNAs and suppresses colon cancer metastasis (Yu et al., 2020). Notably, RBPs might be involved in spermatogenesis and TGCT. DAZL knockout in mouse germ cells leads to complete male sterility through the gradual loss of spermatogonial stem cells (SSCs), meiotic arrest, and spermatid arrest (Li et al., 2019). KHDRBS1 downregulation inhibits the proliferation, accelerates the apoptosis of germ cell, and thus causes spermatogenic defects in human testes (Li et al., 2014). The increased expression of LINC00162 in the nucleus could promote the proliferation of testicular embryonal carcinoma cells via miR-320a and miR-383 by binding to nucleolin, a kind of RBP (Lü et al., 2015). However, few researchers had focused on the prognostic value of RBPs in TGCT; therefore, our work is necessary.

In the current study, we explored the prognostic importance of RBPs in TGCT by analyzing the expression data of differentially expressed RBPs in TGCT obtained from online databases. The risk model constructed by Cox regression analyses and Lasso regression showed that expression of six RBPs is correlated with disease-free survival (DFS) in patients with TGCT. We assessed the specificity and sensitivity of 10-year survival status prediction using receiver operating characteristic (ROC) curve analysis, and successfully validated the results in multiple cohorts. Functional analysis was carried out to seek the possible mechanisms and pathways. We also examined the association between the model based on six RBPs and different clinical characteristics. A TGCT nomogram was established for recurrence prediction. Consensus clustering analysis was carried out to identify the clusters of TGCT with different clinical outcomes.

\section{MATERIALS AND METHODS}

\section{Patient Selection and Data Collection}

The expression data and clinical information of RBPs in 156 patients with TGCT were downloaded from The Cancer Genome Atlas (TCGA) datasets, ${ }^{1}$ and the data of 165 healthy controls were obtained from Genotype-Tissue Expression $\left(\right.$ GTEx). ${ }^{2}$ We downloaded "TCGA-TGCT.survival.tsv.gz" and "TCGA-TGCT.htseq_fpkm.tsv.gz" from TCGA datasets. We further downloaded "gtex_RSEM_gene_fpkm.gz" and "GTEX_phenotype.gz" from the GTEx dataset and selected patients whose "primary site" is "Testis." Patients with Patient data included a complete RBP expression profile and survival data for DFS. This study complied with TCGA publication guidelines and policies. No ethics approval was demanded for this study because data were obtained from online public resources.

In this study, we selected 1,542 RBPs for further analysis (Gerstberger et al., 2014). Adjusted false discovery rate $($ FDR $)<0.05$ and absolute $\mid \log 2$ fold change $>0.5$ were chosen as the cut-off threshold. "Limma" package (3.46.0) was used for screening the differentially expressed genes (DEGs) between cancer samples and normal control samples.

\section{Protein-Protein Interaction (PPI) Network Construction and Module Analysis}

In the current study, information on the protein-protein interaction (PPI) network of RBPs were obtained from the Search Tool for the Retrieval of Interacting Genes (STRING) database ${ }^{3}$ with interaction score $>0.7$, and 388 differentially expressed RBPs were chosen to constitute the PPI network. Plug-in Molecular Complex Detection (MCODE) was applied to detect densely connected regions in PPI networks, which was visualized by Cytoscape (version 3.8.0). ${ }^{4}$ The PPI networks were constructed using Cytoscape and the most significant module in the PPI networks was selected using MCODE. The criteria for selection were set as follows: maximum depth $=100$, degree cut-off $=2$, node score cut-off $=0.2$, MCODE scores $>5$ and K-score $=2$.

\section{Construction of Prognostic Prediction Model}

Our work was designed and executed following the process presented in the flow chart (Figure 1) to find out the roles of RBPs in TCGT. All patients with TGCT in TCGA were randomly divided into two groups. Among 121 patients, 79 were selected as a training cohort and 42 cases were classified into a test cohort. We carried out Cox univariate analysis to identify possible prognostic RBPs based in the training cohort. Then, we excluded some genes highly correlated with one another using

\footnotetext{
${ }^{1}$ http://cancergenome.nih.gov/

${ }^{2}$ https://www.gtexportal.org/home/datasets

${ }^{3} \mathrm{http}: / /$ string-db.org/

${ }^{4}$ https://cytoscape.org/
} 


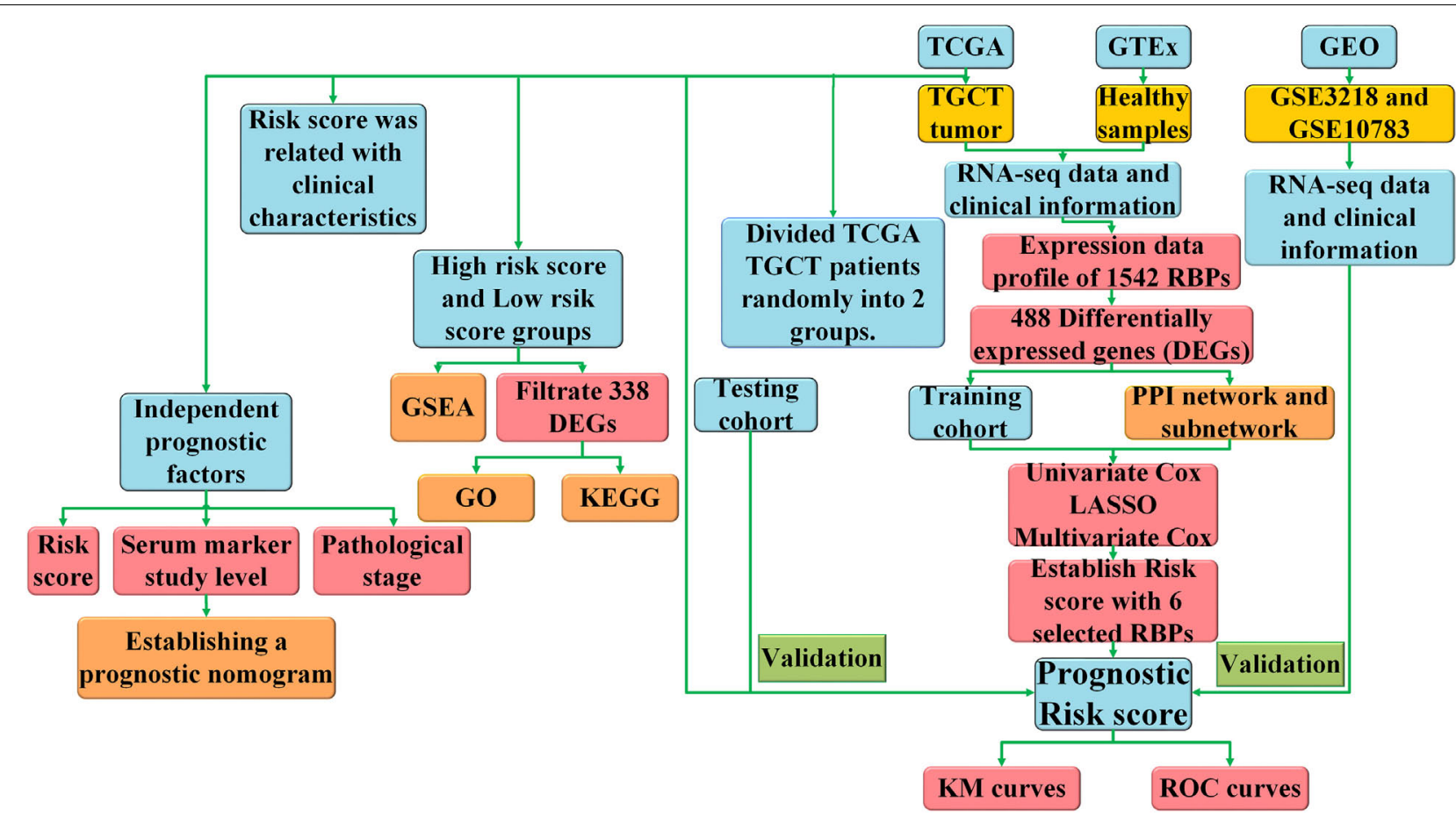

FIGURE 1 | The flow chart of the study design and analysis.

least absolute shrinkage and selection operator (LASSO) Cox regression algorithm to avoid overfitting the model. Ten RBPs were selected for further Cox multivariate proportional hazards regression analysis. Finally, six RBPs (TRMT61A, POLR2J, DIS3L2, IFIH1, IGHMBP2, and NPM2) were identified for the construction of risk score. Risk score was calculated through the following formula: Risk score $=\sum_{i=1}^{n} \operatorname{coef}(i) \times x(i)$ where $n$, $\operatorname{coef}(i)$, and $x(i)$ represent the number of genes, the coefficient of each gene, and the relative expression value of each gene selected by multivariate analysis, respectively. Patients were divided into high-risk and low-risk groups based on the median risk score of all samples. Kaplan-Meier curve and log-rank test were carried out to evaluate the relationship between DFS and risk score. The "glmnet," "survival," and "survminer" packages in R were used to construct the prognostic prediction model.

\section{Validation of Prognostic Signature}

Patients in the test cohort were classified into high-risk and low-risk groups based on the same cut-off risk score. KaplanMeier curve and log-rank test were carried out to evaluate the relationship between DFS and risk score. Then, we validated the prediction accuracy of the prognostic model in the entire TCGA cohort. Kaplan-Meier curve and ROC were carried out. Chi-square test was performed to evaluate the association between clinicopathological parameters and the risk score in the entire TCGA cohort. Univariate and multivariate Cox regression analyses were used in the entire TCGA cohort to investigate whether risk score was an independent prognostic factor. The prognostic value of risk score stratified by clinicopathological parameters was further assessed. The "survival," "survminer," and "survivalROC" packages in R were used to carry out KaplanMeier and ROC analysis.

\section{Establishment and Validation of Nomogram for Prognosis Prediction}

A nomogram for prognosis prediction was constructed using all independent prognostic factors (risk score, serum marker levels, and pathological stage). Calibration plots were used to investigate the calibration of the nomogram. The "rms", "foreign," and "survival" packages in R were used to establish and validate the nomogram.

\section{Functional Analysis}

After dividing the patients with TGCT into two groups based on six-RBP risk score, we subsequently performed Gene Ontology (GO) and Kyoto Encyclopedia of Genes and Genomes (KEGG) pathway enrichment analyses of DEGs between the two groups using the "clusterProfiler" $\mathrm{R}$ package to extract the information of the enriched functions and pathways of the prognostic model based on six RBPs. GO analysis consists of three terms: biological process (BP), cellular component (CC), and molecular function (MF). FDR $<0.05$ and $|\log 2 \mathrm{FC}|>0.5$ were used as thresholds. Gene set enrichment analysis (GSEA) is a systematic method used to figure out whether hallmark gene sets predicted have statistically significant differences between two different groups (Subramanian et al., 2005). We carried out GSEA to analyze significant differences between the survival of the high-risk and 
low-risk groups in the entire TCGA cohort. Normalized $p<0.05$ and FDR $<0.25$ were considered significant differences.

\section{Consensus Clustering Analysis}

The TGCT cohort was clustered into two groups according to the consensus expression of six selected RBPs using "Consensus Cluster Plus" in R to check out whether the expression levels of RBPs have prognostic value. Chi-square test was used to compare the distribution of serum markers, lymphovascular invasion, pathological stage, TMN stage, type, and age between two clusters. We carried out principal component analysis (PCA) to compare the transcriptional profile between clusters 1 and 2 .

\section{External Validations of the Six-Gene Risk Score Using the Gene Expression Omnibus Database}

The six-gene risk score was further verified using two gene expression profiles (GSE3218 and GSE10783) extracted from the Gene Expression Omnibus (GEO) database. ${ }^{5}$ We used the following terms to search the eligible GEO datasets: ("Germ Cell Tumors" or "Testicular Germ Cell Tumor") and ("outcome” or "prognosis") and ("male"). The eligible studies met the following inclusion criteria: (Tsili et al., 2019) reported research on patients with TCGT; (Zhang et al., 2018) provided sufficient clinical data to calculate overall survival (OS), DFS, or progression-free survival; and (Diamantopoulos and Kortsaris, 2010) provided gene expression profiles of TGCT. Only peer-reviewed studies were deemed eligible for inclusion. Conference abstracts and case reports were excluded. After searching the databases, the data source of the references was examined to avoid duplicates. Data

${ }^{5}$ https://www.ncbi.nlm.nih.gov/geo/ from the most complete study was extracted when duplicates in study population were found. The "sva" package was used to remove batch effects and other needless variables. Overall, 108 samples from GSE3218 and GSE10783 were used as the independent external validation cohort. Kaplan-Meier curve and log-rank test were carried out to evaluate the relationship between OS and risk score.

\section{Statistical Analysis}

All statistical data and figures were analyzed using R 4.0.0. We assessed the different expression of RBPs between the control and TGCT groups by Wilcoxon's test. Chi-square test was performed to evaluate the association between risk score and clinicopathological parameters. Univariate and multivariate Cox regression analyses were used in the entire TCGA cohort to investigate whether risk score is an independent prognostic factor. ROC curve and the area under the ROC curve (AUC) were used to evaluate the prognostic ability of risk score using the package of "survivalROC" in R. All statistical results with $p<0.05$ were considered statistically significant.

\section{RESULTS}

\section{Differentially Expressed RBPs in TGCT}

We analyzed the expression profiles of RBPs between 156 tumor samples and 165 healthy controls healthy individuals from TCGA and GTEx. We screened 489 DEGs, including 287 significantly up-regulated RBPs and 222 down-regulated RBPs. All the DEGs were identified based on $|\log 2 \mathrm{FC}|>0.5$ and FDR $<0.05$. Heatmap and volcano plot (Figure 2) were also constructed to visualize the expression patterns of DEGs, in which red
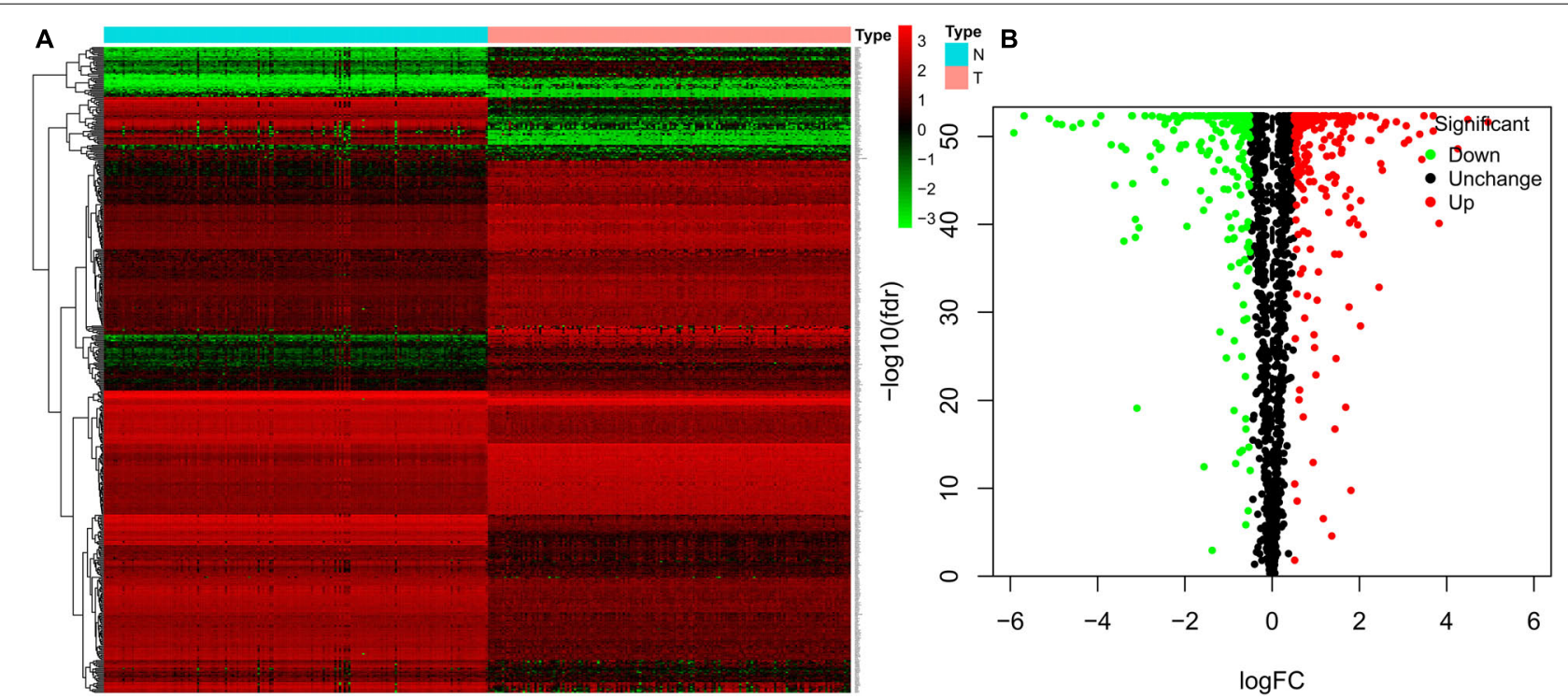

FIGURE 2 | Heatmap and volcano plots of TGCT patients from TCGA. Heatmap (A) and volcano (B) plots were generated with FDR < 0.05 and | log2FC| > 0.5, using the data of differentially expressed RBPs in TGCT downloaded from TCGA and GTEx. TGCT, Testicular germ cell tumors; TCGA, The Cancer Genome Atlas; RBPs, RNA-binding proteins; GTEx, Genotype-Tissue Expression; N, Normal controls from healthy individuals; T, tumor samples of testicular germ cell tumors. 
and green colors represent relatively high and low expression levels, respectively.

\section{PPI Network Among Differentially Expressed RBPs}

We used STRING to establish the PPI network and further explore the correlation and interaction between the differentially expressed RBPs. Cytoscape was also applied to visualize the interaction network. The most meaningful modules were selected and are shown in Figure 3, in which red and green colors meant relatively high and low gene expression, respectively.

\section{Construction of RBP-Related Risk Score in the TCGA Training Cohort and Validation of the Risk Score in the TCGA Test Group}

The DFS data of 132 patients with TGCT were obtained from TCGA and randomly divided into the training and

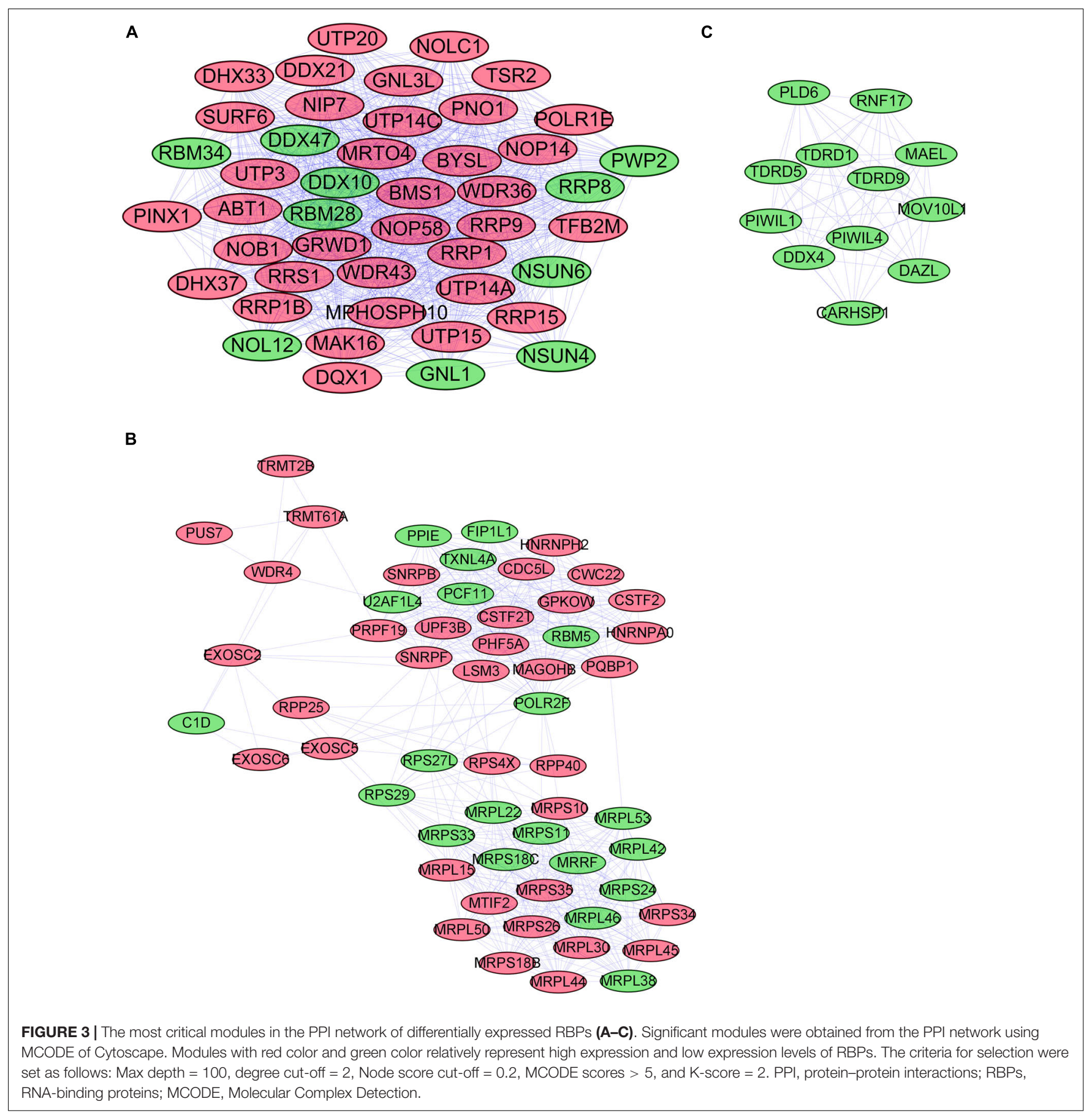




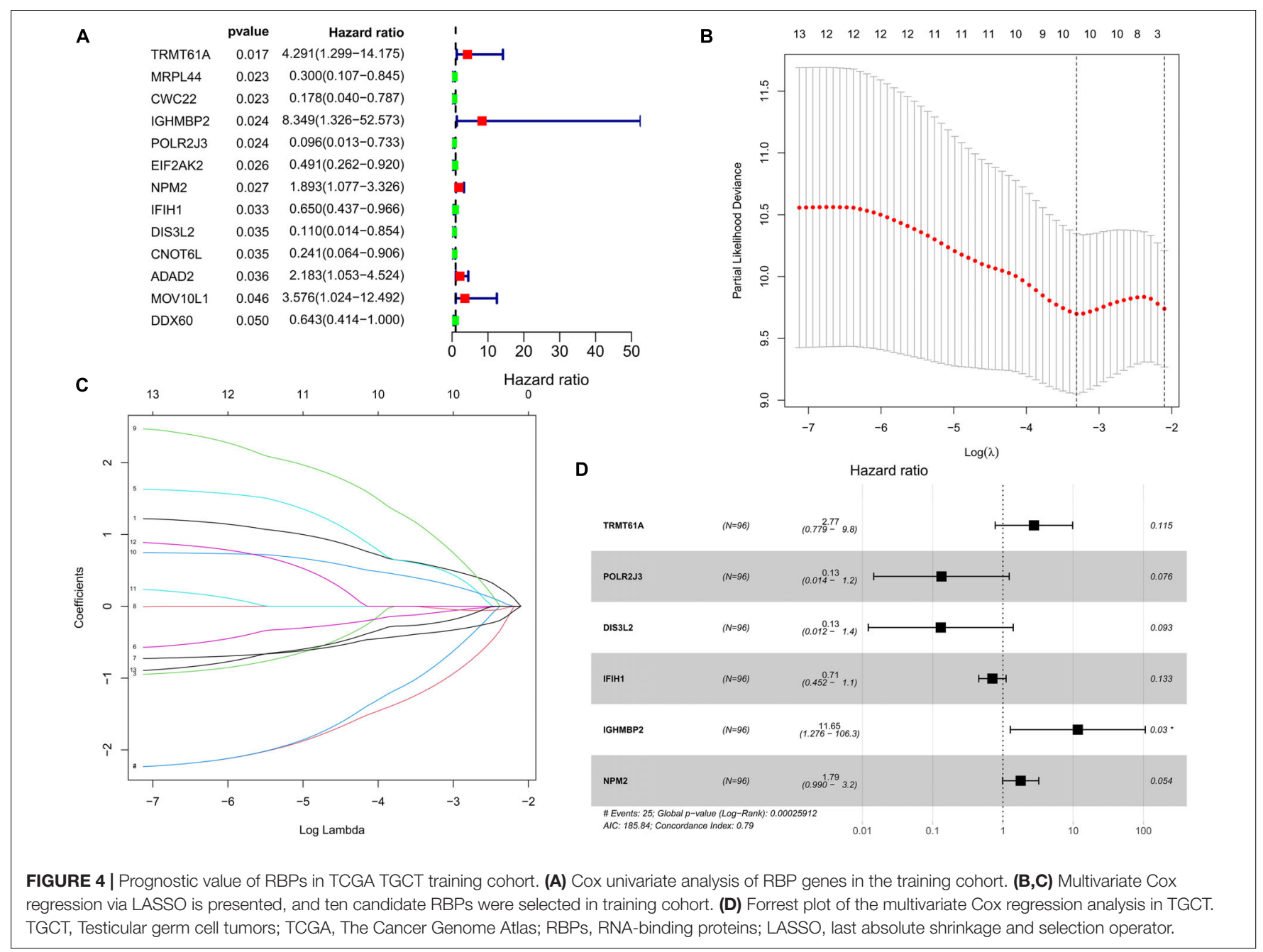

test groups. In the training group, we conducted univariate Cox regression based on DFS and identified 13 genes that strongly indicated TGCT prognosis and were significantly related to DFS ( $p<0.05$, Figure $4 \mathrm{~A}$ ). Then, we used LASSO regression to avoid overfitting (Figures $4 \mathbf{B}, \mathbf{C}$ ). We finally targeted six key genes (TRMT61A, POLR2J, DIS3L2, IFIH1, $I G H M B P 2$, and NPM2) that met the modeling requirement by multivariate Cox regression analysis (Figure 4D). Each gene expression value was matched with its relevant coefficient, and the risk scores of the training cohort were calculated using the formula: Risk score $=($ TRMT61A expression $) \times$ $(1.01815998520062)+($ POLR2J3 expression $) \times(-2.01356263$ $039402)+($ DIS3L2 expression $) \times(-2.03760675664572)$ $+($ IFIH1 expression $) \times(-0.34441879625446)+($ IGHMBP2 expression $) \times(2.45508579120534)+($ NPM2 expression $)$ $\times(0.582848422396182)$. Patients in the training group were subdivided into high- and low-risk groups according to the median risk score. Kaplan-Meier survival curve analysis revealed that the high-risk group has worse prognosis than the low-risk group ( $p<0.001$, Figure 5A). Based on the prognostic model, the survival status or prognosis of patients with TGCT became worse, that is, the number of dead patients increased as the risk score increased (Figures 5B,C). The expression patterns of the six risk RBPs in the high-risk and low-risk groups are shown in the a heatmap in Figure 5D. Time-dependent ROC analysis was applied to assess the predictive efficiency of the model. The AUCs were $0.857,0.802,0.779$, and 0.749 at $1,3,5$, and 10 years, respectively (Figures $5 \mathrm{E}-\mathbf{H}$ ). The results indicated that the model is sensitive and has fair accurance for prognosis prediction. We validated the prognostic models in the test group and proved that the model has the same predictive function as that in the training group (Figure 6). In the test group, the high-risk group has worse prognosis compared with the low-risk group in Kaplan-Meier survival curve analysis $(p<0.01$, Figure 6A). Time-dependent ROC curve was made, and the AUCs were 0.762 at 1 years, 0.857 at 3 and 5 years, and 0.643 at 10 years (Figures 6E-H).

\section{Validation of Risk Score in the Entire TCGA Cohort}

We proved that risk score is valuable in TGCT prognosis prediction in the train and test groups. Next, the model was validated using the entire TCGA cohort. The Kaplan-Meier plots reveal that patients in the high-risk groups exhibited 
A

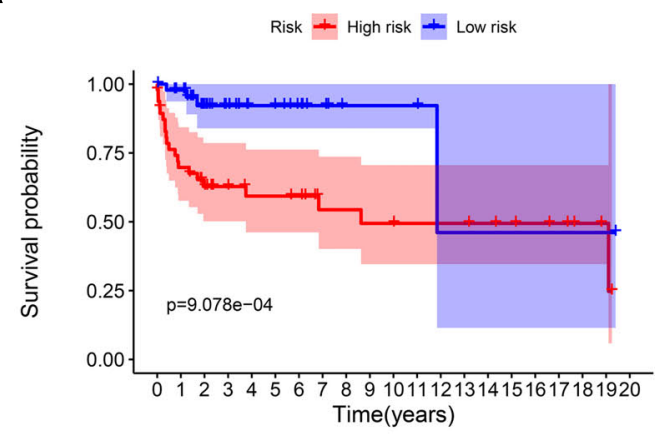

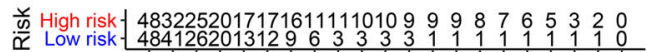
01234567891011121314151617181920
01120

B

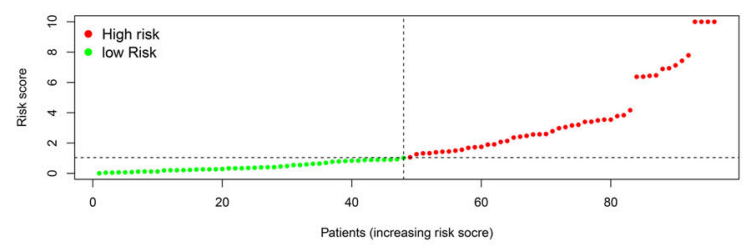

C

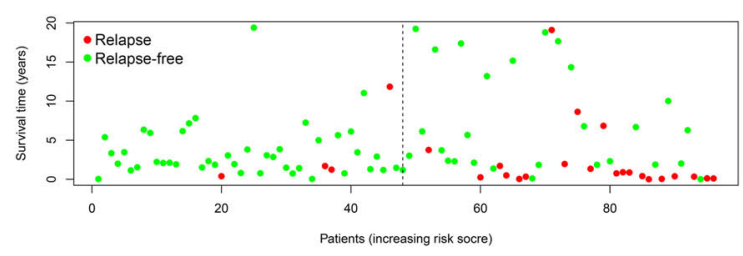

D

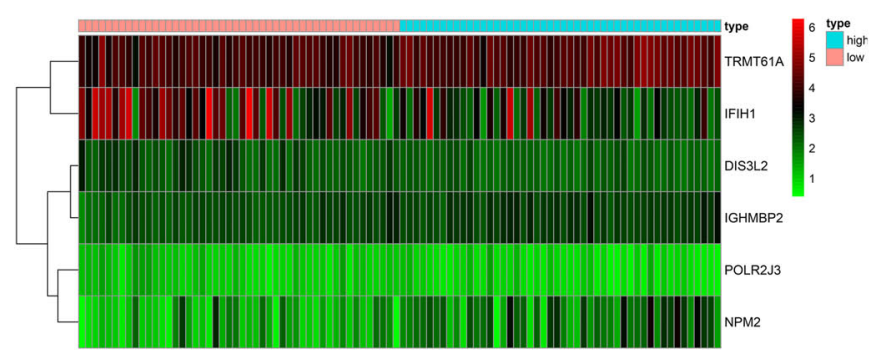

E

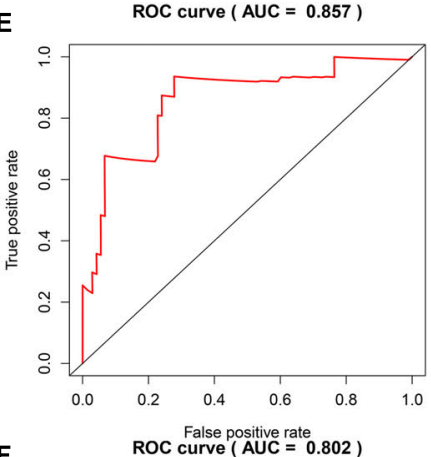

F

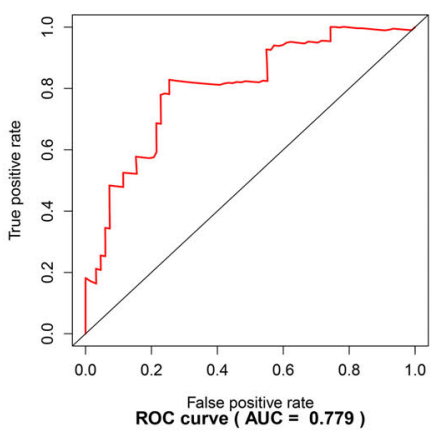

G
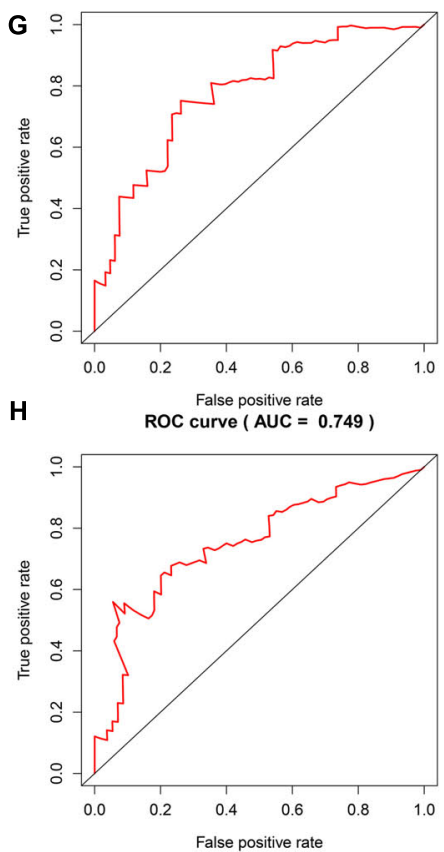

FIGURE 5 | Survival analysis based on the risk model in the training group. (A) Kaplan-Meier survival curve analysis of DFS in the high-risk and low-risk TGCT patients in the training group, marked as red line and blue line separately. (B-D) Risk score, distribution of survival status between the high-risk and low-risk groups, and expression heat maps of six RBPs. (E-H) Time-dependent ROC curve analyses was conducted and AUC values were calculated for 1-, 3-, 5-, and 10-year DFS in the TGCT training cohort. TGCT, Testicular germ cell tumors; RBPs, RNA-binding proteins; DFS, disease-free survival; ROC, Receiver operating characteristic curve; AUC, the area under the ROC curve; High, high-risk group; Low, low-risk group.

worse prognosis than those in the low-risk group $(p<0.001$, Figure 7A). The patients with TGCT from the entire TCGA cohort have worse outcomes as indicated by the increased number of relapsing patients as the risk score increased (Figures $\mathbf{7 B}, \mathbf{C}$ ). The heatmap of the six key genes shows that the expression of TRMT61A is high in the high-risk and low-risk group, whereas DIS3L2, IGHMBP2, POLR2J3, and NPM2 have low expression in the two groups (Figure 7D). IFIH1 has relatively lower expression in the high-risk group than in the low-risk group. Time-dependent ROC curve was 


\section{A}

Risk — High risk + Low risk
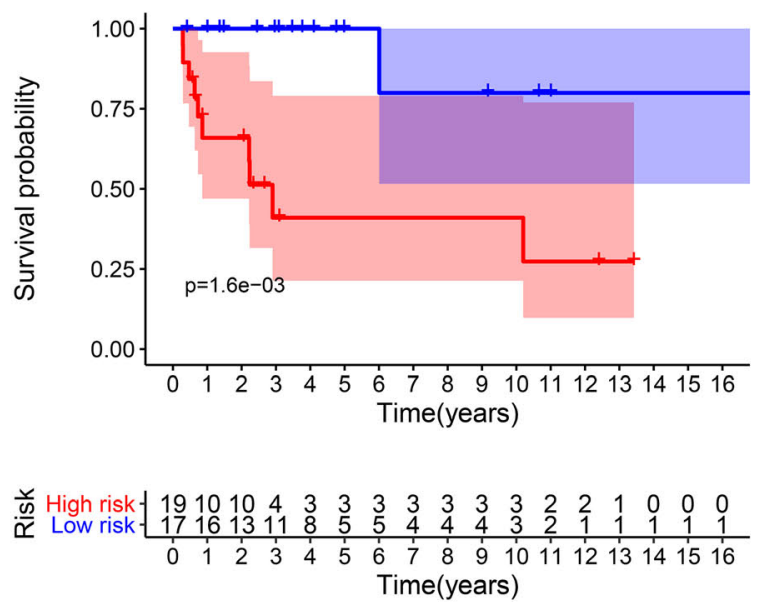

B

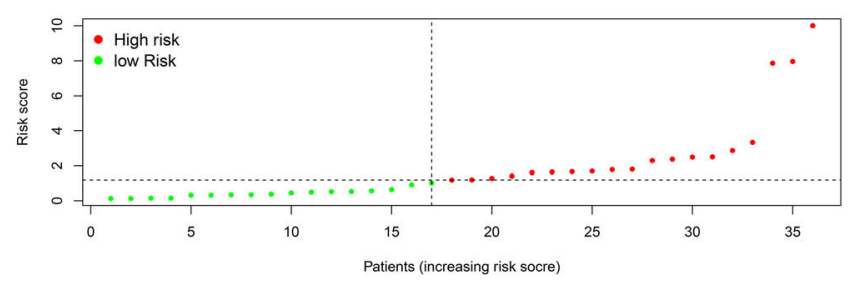

C

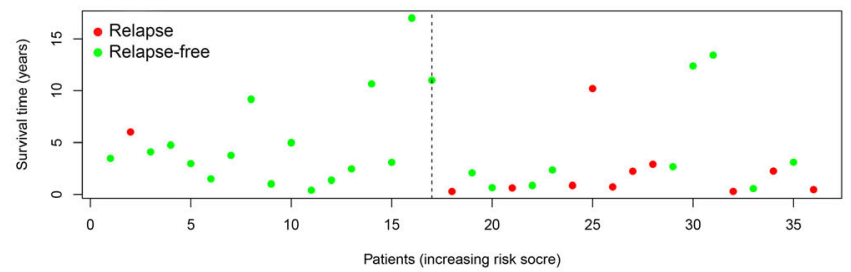

D

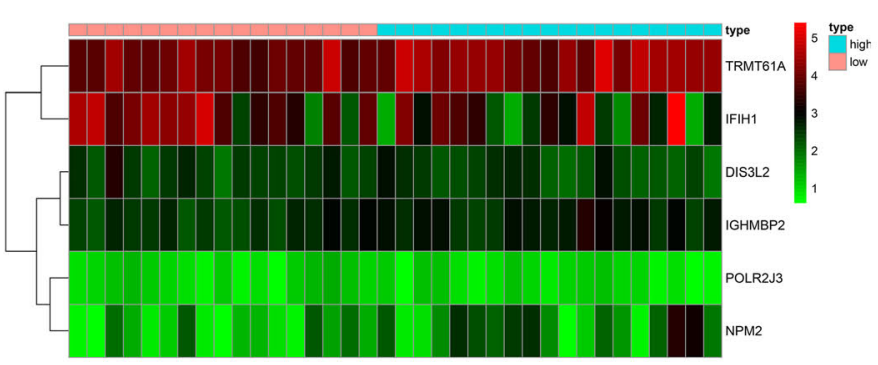

E

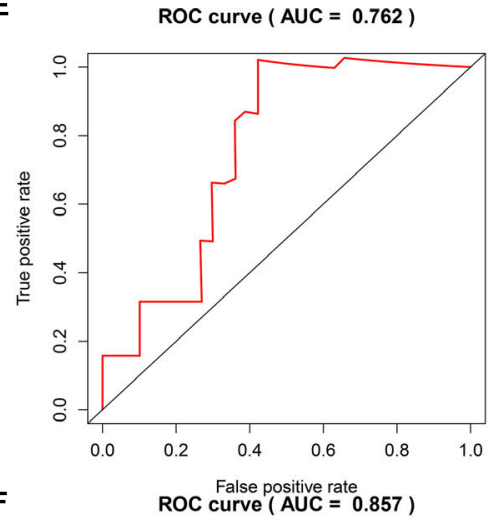

F

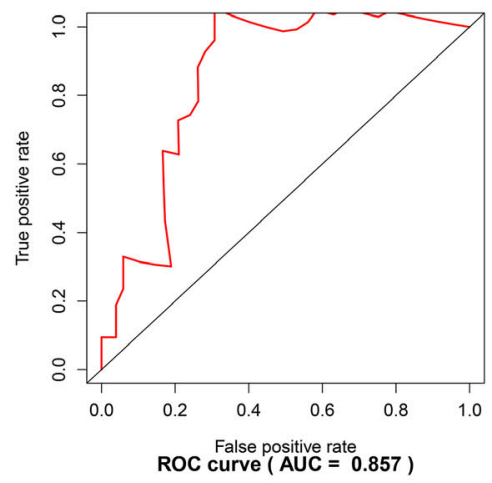

G

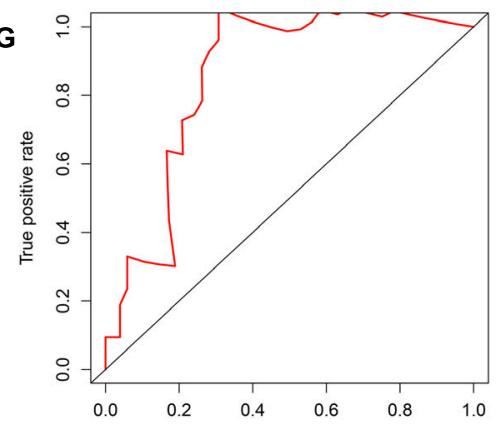

H

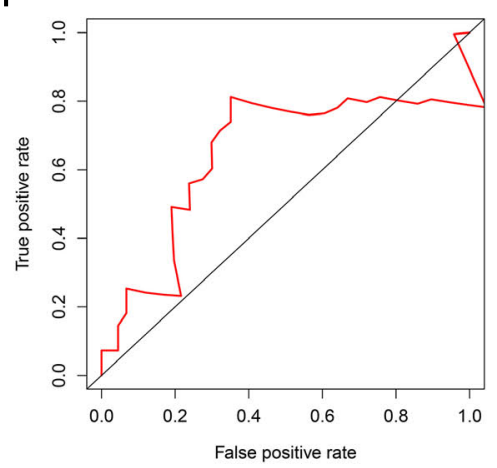

FIGURE 6 | Validation of the prognostic value of the risk model in the test group. (A) Kaplan-Meier survival curve analysis of DFS in the high-risk and low-risk TGCT patients in the test group, marked as red line and blue line separately. (B-D) Risk score, distribution of survival status between the high-risk and low-risk groups and expression heat maps of six RBPs. (E-H) Time-dependent ROC curve analyses was conducted and AUC values were calculated for 1-, 3-, 5-, and 10-year DFS in the TGCT test cohort. TGCT, Testicular germ cell tumors; DFS, disease-free survival; RBPs, RNA-binding proteins; TGCT, Testicular germ cell tumors; ROC, Receiver operating characteristic curve; AUC, the area under the ROC curve; High, high-risk group; Low, low-risk group. 
A
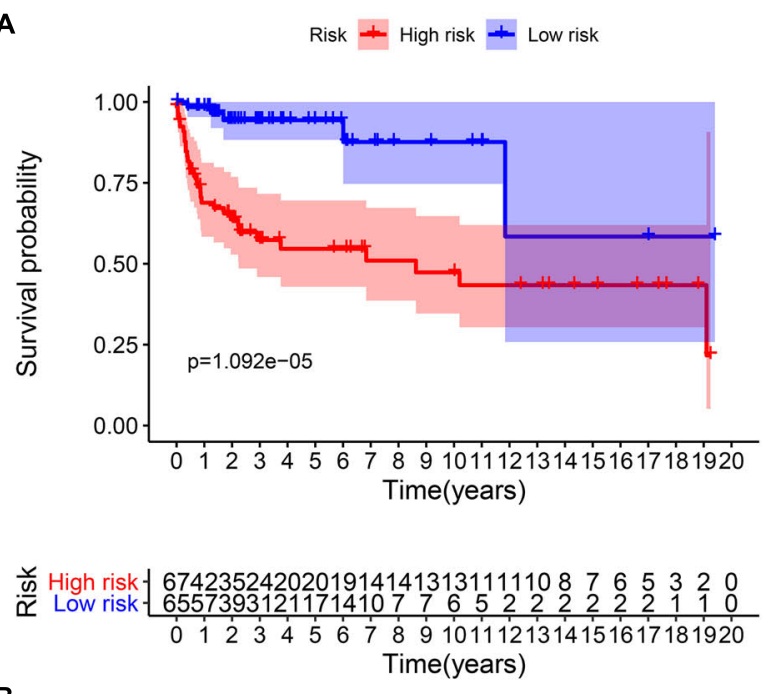

B

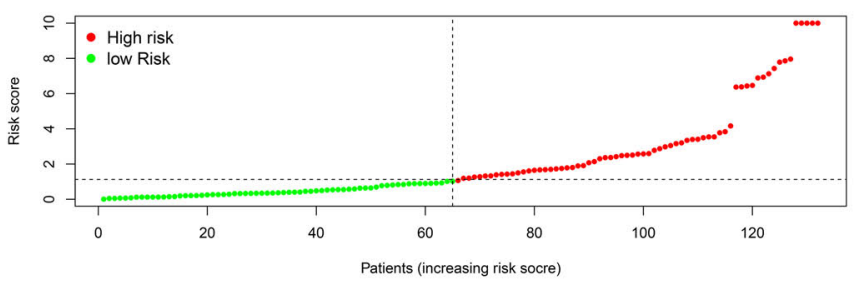

C

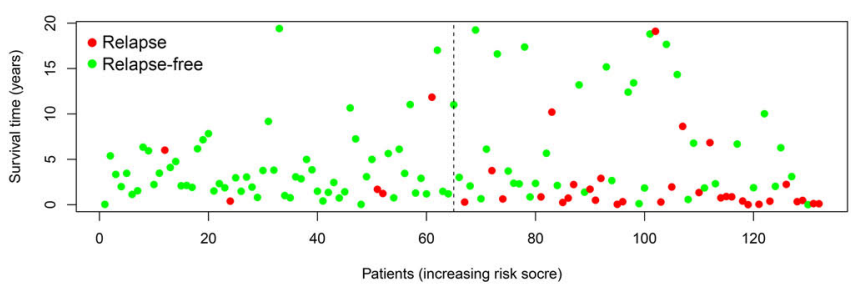

D

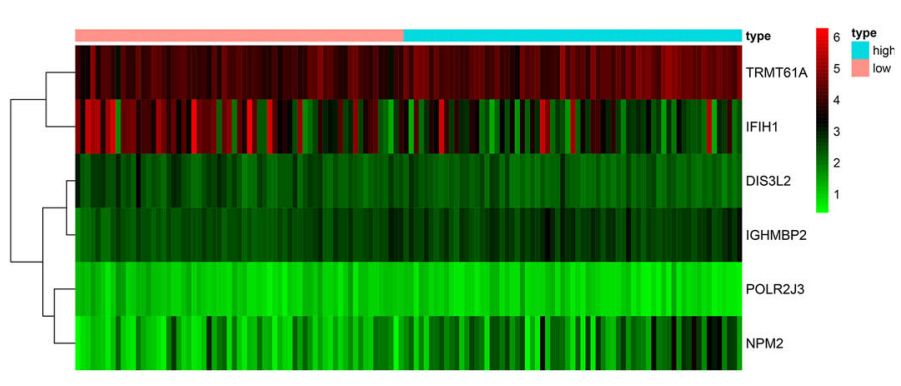

E

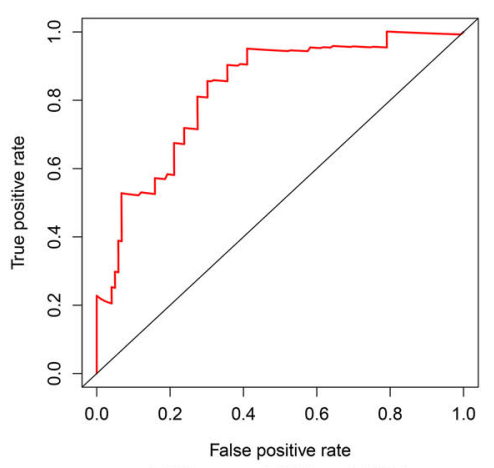

$\mathbf{F}$

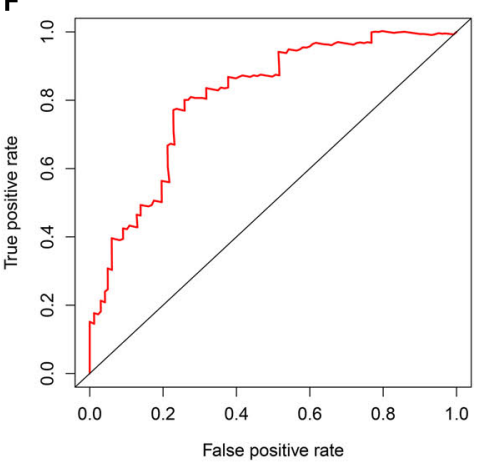

ROC curve $(A \cup C=0.795)$

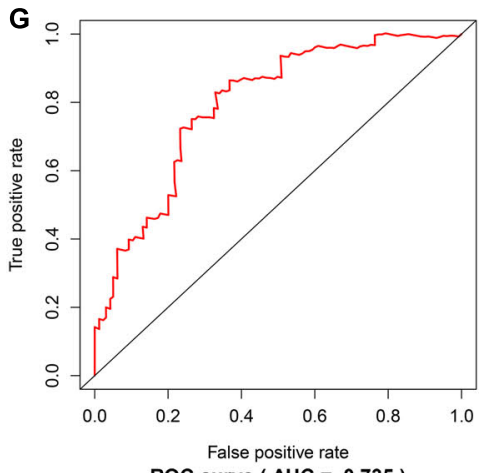

H

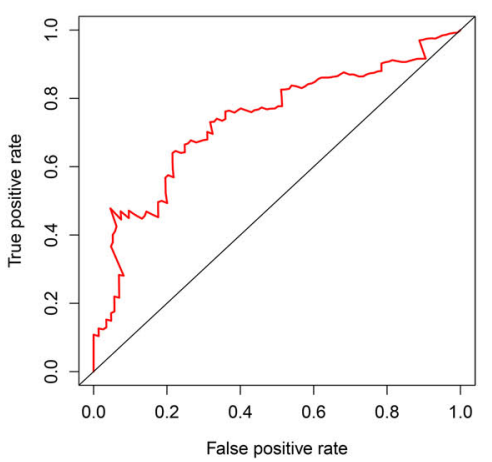

FIGURE 7 | Validation of the prognostic value of the risk model in the entire TCGA cohort. (A) Kaplan-Meier survival curve analysis of DFS in the high-risk and low-risk TGCT patients in the entire TCGA cohort, marked as red line and blue line separately. (B-D) Risk score, distribution of survival status between the high-risk and low-risk groups, and expression heat maps of six RBPs. (E-H) Time-dependent ROC curve analyses was conducted and AUC values were calculated for 1-, 3-, 5-, and 10-year DFS in the entire TCGA TGCT cohort. TGCT, Testicular germ cell tumors; TCGA, The Cancer Genome Atlas; DFS, disease-free survival; ROC, Receiver operating characteristic curve; AUC, the area under the ROC curve; TGCT, Testicular germ cell tumors. 
constructed, and the AUCs were 0.828 at 1 years, 0.808 at 3 years, 0.795 at 5 years, and 0.735 at 10 years (Figures $7 \mathbf{E}-\mathbf{H}$ ). Meanwhile, we also observed that comparing with each gene alone, the six genes-based risk score showed better prognostic value in 1-year (AUC $=0.828$, Supplementary Figure 1A), 3-year $(A U C=0.808$, Supplementary Figure 1B), 5-year (AUC 0.795, Supplementary Figure 1C), and 10-year DFS prediction $(\mathrm{AUC}=0.735$, Supplementary Figure 1D).

\section{Prognostic Signature-Based Risk Score Was an Independent Prognostic Factor in the TCGA TGCT Cohort}

Patient information, such as risk score, age, stage based on serum marker study levels, lymphovascular invasion, TNM stage, pathological stage, and pathological type, were extracted. Univariate and multivariate Cox regression analysis were conducted. The results presented as forest maps obviously indicate that the risk score correlated independently with DFS in univariate [hazard ratio $(\mathrm{HR})=1.155$, confidence interval $\left.(\mathrm{CI})=1.086-1.228,{ }^{* * *} p<0.001\right]$, and multivariate Cox regression analyses $(\mathrm{HR}=1.226,95 \% \mathrm{CI}=1.132-1.327$, ${ }^{* * *} p<0.001$; Figures 8A,B). Stage based on serum markers is also an independent predictor as validated by univariate $\left(\mathrm{HR}=1.802,95 \% \mathrm{CI}=1.179-2.756,{ }^{* *} p=0.007\right)$ and multivariate Cox regression analyses $(\mathrm{HR}=3.563,95 \%$ $\left.\mathrm{CI}=2.021-6.279,{ }^{* * *} p<0.001\right)$. In addition, pathological stage was validated in multivariate Cox regression analysis $\left(\mathrm{HR}=0.115,95 \% \mathrm{CI}[0.024-0.558],{ }^{* *} p=0.007\right)$. The heat map of the expression levels of the six key RBPs in the high- and low-risk groups patients in the TCGA cohort is presented in Figure 8C. Clinicopathological parameters, namely, pathological type $\left({ }^{* * *} p<0.001\right)$, pathological stage $\left({ }^{*} p<0.05\right)$, and higher $\mathrm{M}$ stage $\left({ }^{*} p<0.05\right)$, were significantly different between the highand low-risk groups (Figure 8C).

Time-dependent ROCs were applied to assess the prognostic accuracy of factors, including risk score, age, serum marker study levels, lymphovascular invasion, TNM stage, pathological stage, and type in patients with TGCT (Figure 9). Compared with other factors, risk score manifested superior accuracy in predicting 1-year (AUC $=0.779$, Figure 9A), 3-year $(\mathrm{AUC}=0.775$, Figure 9B), 5-year (AUC $=0.775$, Figure 9C), and 10-year survival (AUC $=0.726$, Figure 9D). Stage based on serum marker study levels was only inferior to risk score but also showed great accuracy in predicting 1-year (AUC $=0.747$, Figure 9A), 3-year (AUC $=0.691$, Figure 9B), 5-year $(A U C=0.691$, Figure 9C), and 10 -year survival (AUC $=0.627$, Figure 9D).

\section{The Survival Difference Between High- and Low-Risk Group Stratified by Clinicopathological Parameters in the TCGA TGCT Cohort}

We grouped the patients by clinical characteristics to figure out whether risk score has prognostic value in various clinicopathological parameters. Kaplan-Meier curves were constructed to show that patients in the high-risk group with clinical characteristics, such as age $\geq 36$ ( $p=0.009$, Figure 10A), age $\leq 36(p<0.001$, Figure 10B), no lymphovascular invasion $(p=0.012$, Figure 10C), lymphovascular invasion $(p=0.004$, Figure 10D), serum tumor marker levels within normal limits $(p=0.006$, Figure 10E), serum tumor marker levels beyond normal limits $(p=0.004$, Figure 10F), seminoma $(p<0.001$, Figure 10H), T1 stage $(p<0.006$, Figure 10I), T2-3 stage

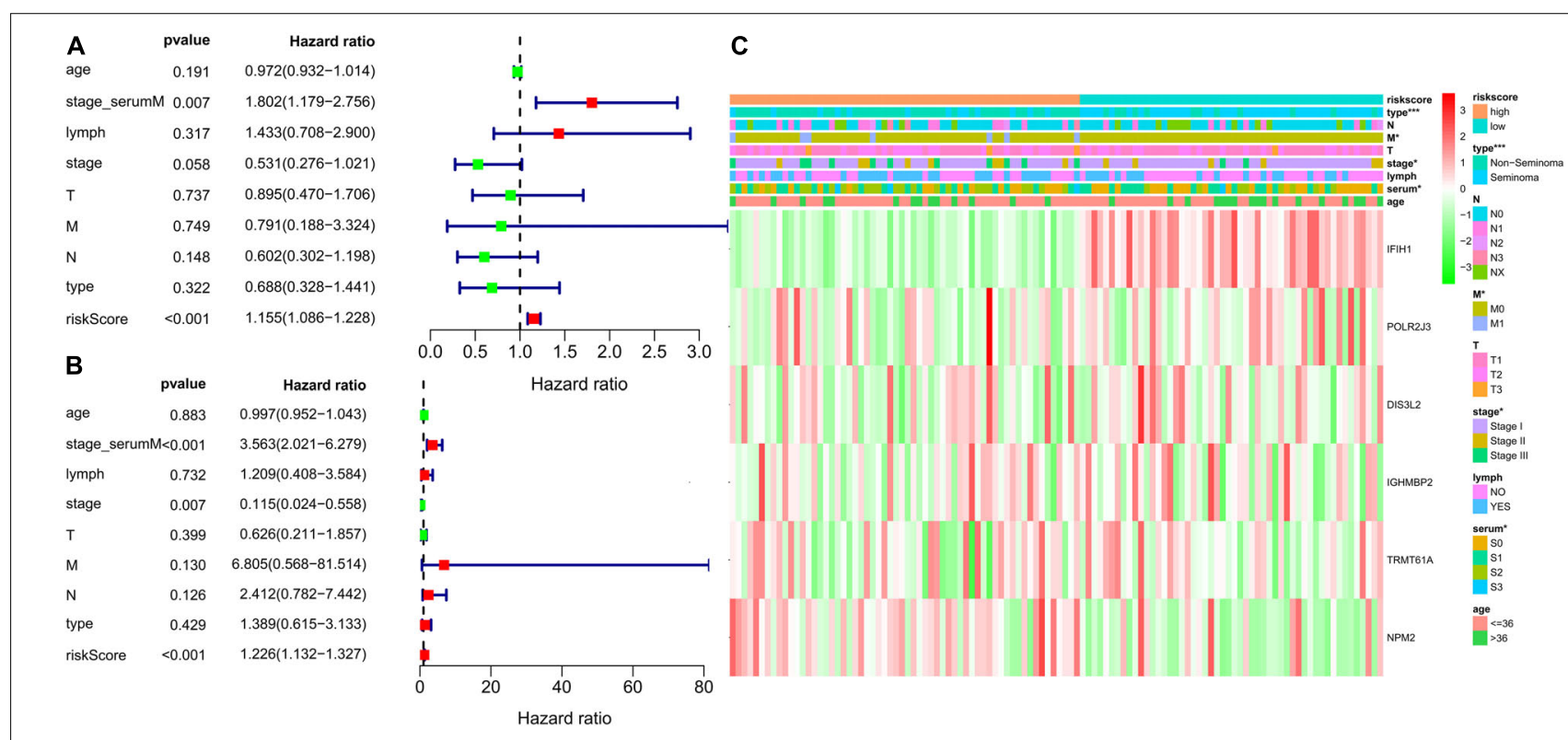

FIGURE 8 | Analysis for evaluating the independent prognostic value of the risk score. Forrest plot of univariate (A) and multivariate (B) Cox regression analysis of risk score, age, serum markers, lymphovascular invasion, TNM stage, disease type, and stage. (C) Significant differences were found for the disease type, M stage, serum markers, and stage between high- and low-risk group. ${ }^{*} p<0.05$, ${ }^{* *} p<0.01$, and ${ }^{* \star *} p<0.001$ compared with the low-risk group. 


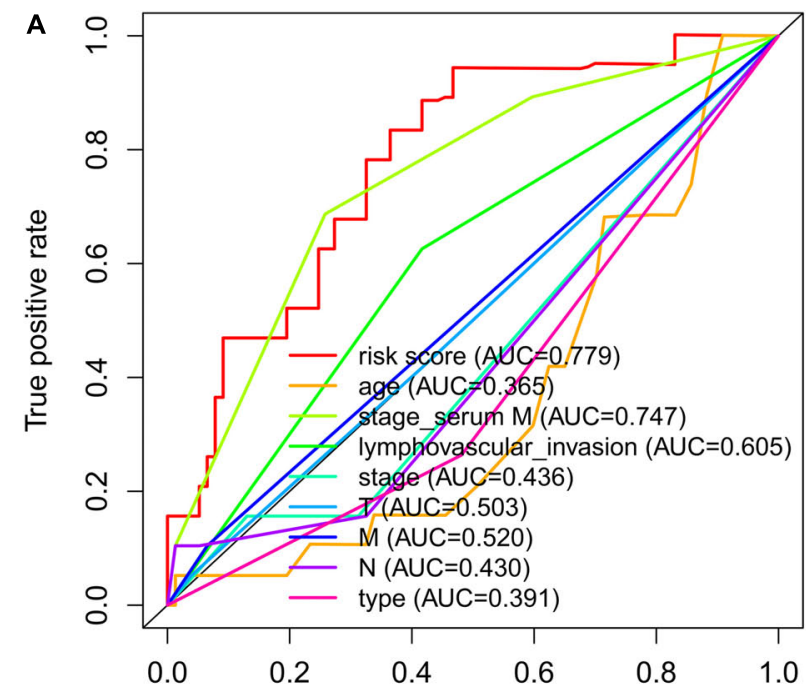

C

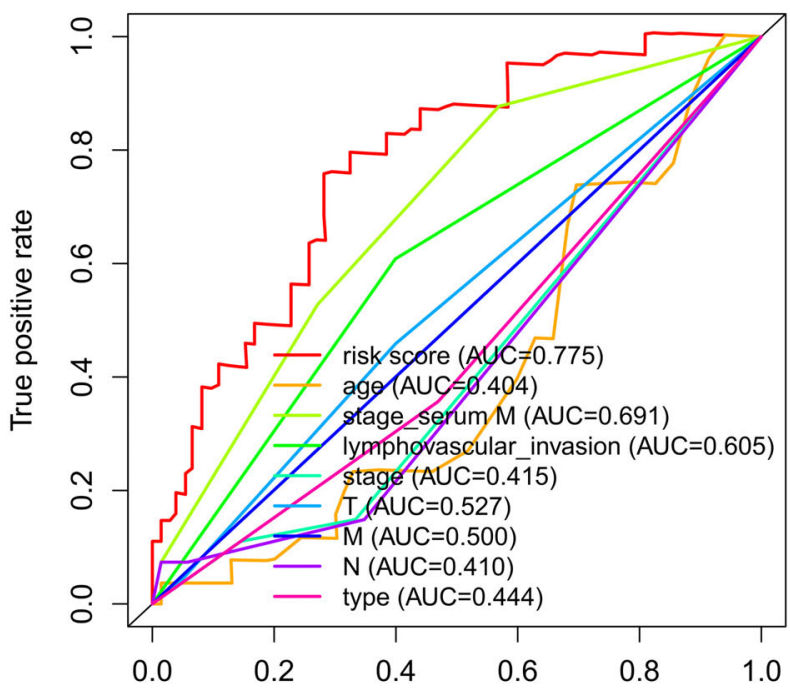

False positive rate

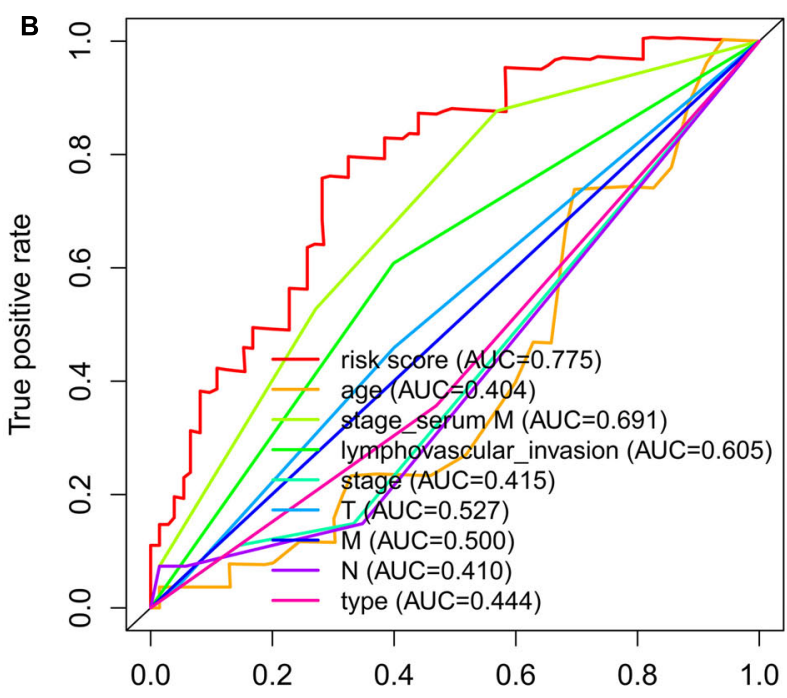

D

False positive rate

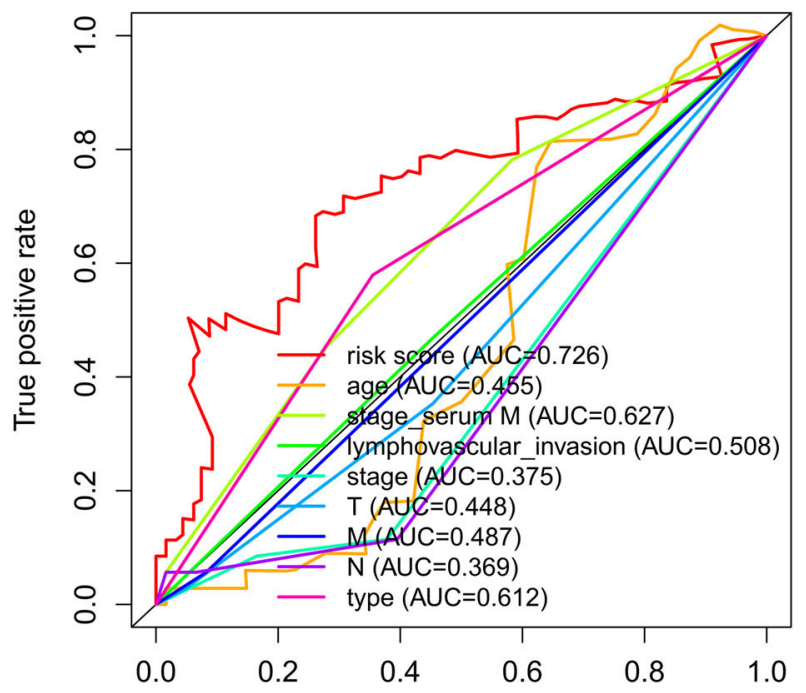

False positive rate

FIGURE 9 | The time-dependent ROC curves for risk score, age, serum markers, lymphovascular invasion, TNM stage, disease type, and stage combining with 1 (A), 3- (B), 5- (C), and 10- (D) year DFS in TCGA TGCT cohort, respectively. DFS, disease-free survival; ROC, Receiver operating characteristic curve; TCGA, The Cancer Genome Atlas; TCGA, The Cancer Genome Atlas; DFS, disease-free survival; ROC, Receiver operating characteristic curve; TGCT, Testicular germ cell tumors.

$(p=0.001$, Figure 10J), M0 stage $(p<0.001$, Figure 10K), had significantly lower DFS rate than those in the low-risk group. However, no significant difference was found in patients diagnosed with non-seminoma ( $p<0.098$, Figure 10G) and M1 stage $(p=0.246$, Figure 10L).

The Kaplan-Meier plots of the risk scores based on the six RBPs are exhibited in Figure 11. The patients with TCGT in the low-risk group who had high DIS3L2, IFIH1, and POLR2J3 expression and low TRMT61A, IGHMBP2, and NPM2 expression presented better prognosis than those in the highrisk group, and differences were all statistically significant.
Therefore, these six genes could play a prognostic role in TCGT prognosis prediction.

\section{Establishment of TGCT DFS Prediction Nomogram}

We established the nomogram predictive model to better predict the probability of disease progression by integrating independent factors associated with prognosis (serum marker study levels, stage, and risk score; Figure 12A). We could predict the 1-year (Figure 12B), 3-year (Figure 12C), and 


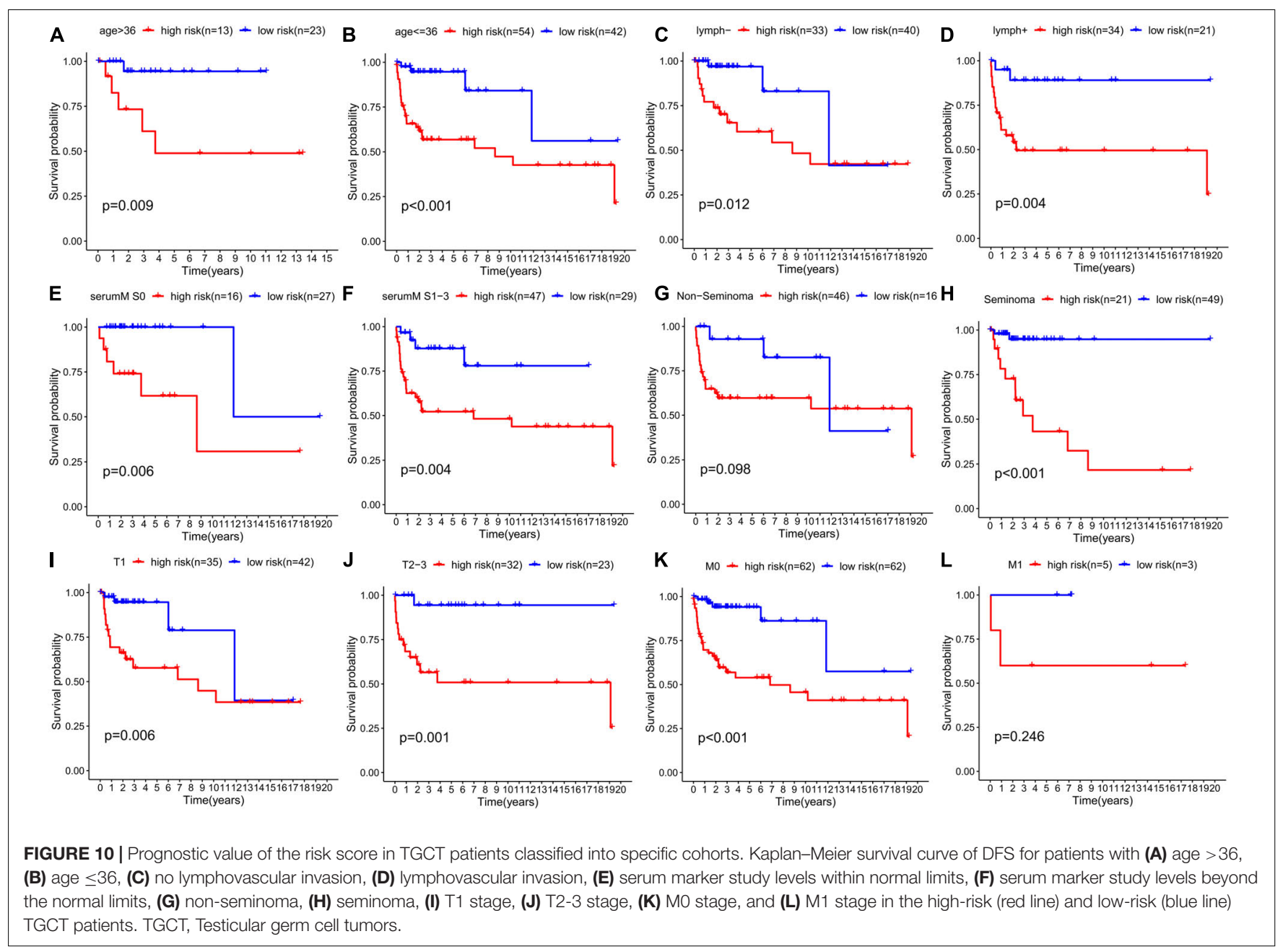

5-year (Figure 12D) survival rates of TGCT patients respectively according to the total points of all risk factors by quantifying the variables above as numeric scores and adding them up. We also constructed calibration plots to manifest that the predicted outcomes were consistent with the observed outcomes. The performance of the nomogram in accurately predicting the 1-, 3 -, and 5-year survival of diagnosed patients was proven in this study.

\section{Identification of Signaling Pathways Related to Risk Score by GO, KEGG, and GSEA}

We divided the 132 patients into high-risk and low-risk groups based on risk score and identified 338 DEGs based on the criteria $|\log 2 \mathrm{FC}|>1$ and FDR $<0.05$. We aimed to illuminate the biological functions and pathways related to DEGs between highrisk and low-risk groups by performing GO and KEGG pathway enrichment analyses. As illustrated in Figure 13A, The ten most significant BP terms related with the up-regulated RBPs include "negative regulation of growth," "somatic stem cell population maintenance," "response to zinc ion," "detoxification of copper ion," "stress response to copper ion," "stress response to metal ion," "cellular response to zinc ion," "cellular response to copper ion," and "cellular zinc ion homeostasis." In Figure 13B, the 10 most significant BP terms related with down-regulated RBPs include "humoral immune response," "complement activation, classical pathway," "humoral immune response mediated by circulating immunoglobulin," "complement activation," "protein activation cascade," "immunoglobulin mediated immune response," and "B cell mediated immunity." Likewise, GO CC terms "cell-cell junction," "collagen-containing extracellular matrix," "endoplasmic reticulum lumen," "membrane raft," "membrane microdomain," "immunoglobulin complex," "immunoglobulin complex, circulating," "external side of plasma membrane," and "blood microparticle" were enriched. GO MF terms revealed that highly expressed RBPs were enriched in "receptor ligand activity," "cell adhesion molecule binding," "growth factor activity," "peptidase regulator activity," "cadherin binding," "peptidase inhibitor activity," "cytokine activity," "transforming growth factor beta receptor binding," and "cadherin binding involved in cell-cell adhesion," and the lowly expressed RBPs were enriched in "antigen binding" and "immunoglobulin receptor binding." 


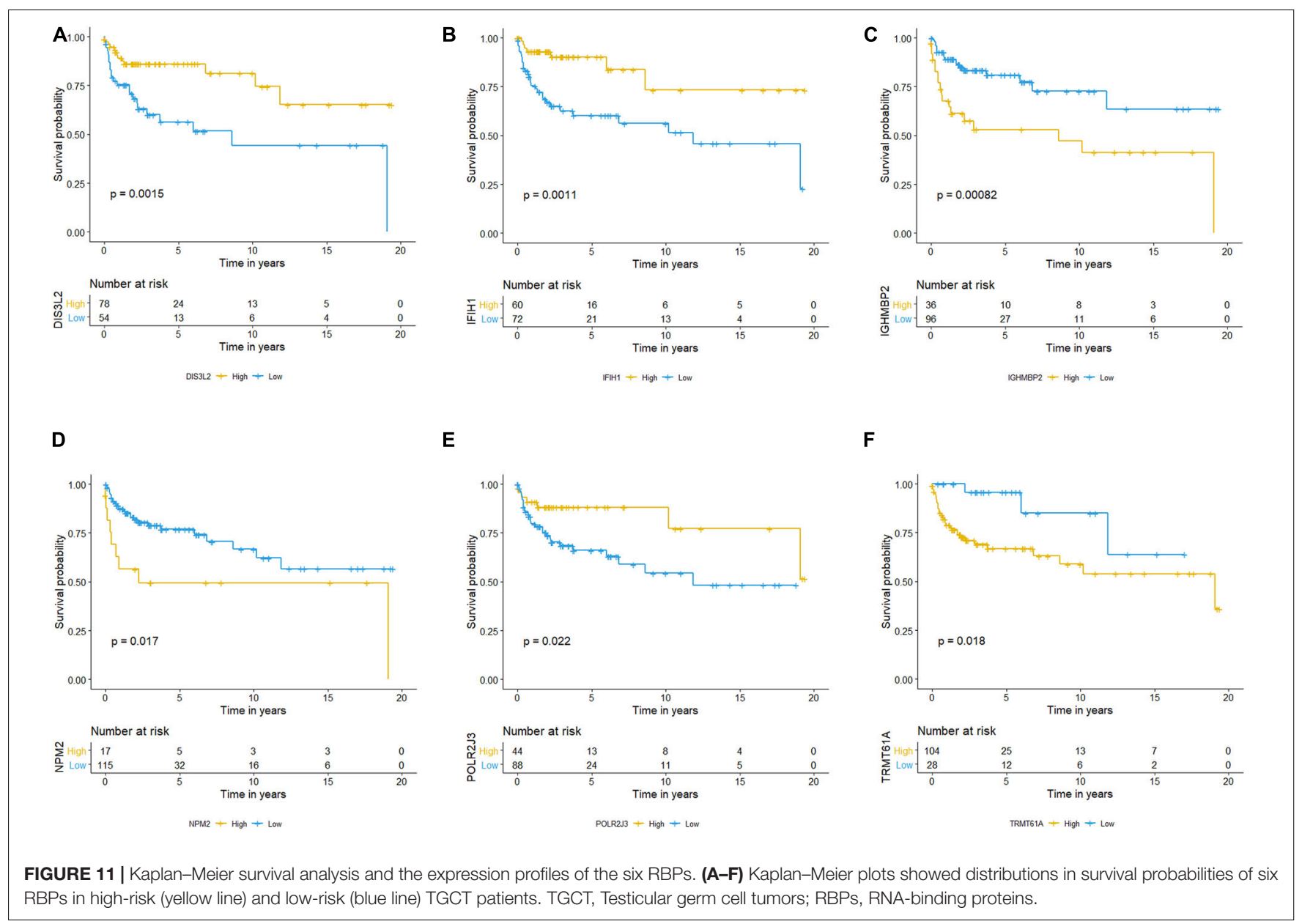

The significant pathways related with the DEGs listed in Figures 13C,D were analyzed by KEGG. The pathways include "signaling pathways regulating pluripotency of stem cells," "cytokine-cytokine receptor interaction," "mineral absorption," "melanoma," "proteoglycans in cancer," "biosynthesis of amino acids," "TGF-beta signaling pathway," "breast cancer," "HIF1 signaling pathway," Pathways such as "phospholipase D signaling pathway," "Wnt signaling pathway," "Ras signaling pathway," "arachidonic acid metabolism," and "protein digestion and absorption" were negatively related to risk score.

Gene set enrichment analysis was performed to further investigate the possible mechanisms leading to different outcomes in the high-risk group and the low-risk group, and we did GSEA based on the patients in two groups (Figure 13E). "Alanine aspartate and glutamate metabolism," "amino sugar and nucleotide sugar metabolism," "cysteine and methionine metabolism," "glutathione metabolism," "glycine serine and threonine metabolism," "glycolysis gluconeogenesis," "oxidative phosphorylation," "p53 signaling pathway," "proteasome," and "spliceosome" were the enriched pathways in the high-risk group. The results of GSEA demonstrated that most of the DEGs in the high-risk group were enriched in cancer pathways and metabolism related pathways.

\section{Consensus Clustering of Risk Score Based on Six Independent Prognostic RBPs Identified Two Clusters of TGCT With Different Clinical Outcomes}

We selected $\mathrm{k}=2$ as the most appropriate selection to divide the TGCT patient cohort into two clusters, namely, cluster 1 and cluster 2, according to the expression similarity of risk score based on independent prognostic RBPs and the criteria for selecting the number of clusters (Figures 14A-C). PCA was carried out to compare the transcriptional profiles of clusters 1 and 2, and substantial distinction was observed between the two subgroups (Figure 14D). The assessment of the correlation between the clusters and clinicopathological features showed significant differences in type $\left({ }^{* * *} p<0.001\right)$, M stage $\left({ }^{*} p<0.05\right)$, pathological stage $\left({ }^{*} p<0.05\right)$, and serum markers study levels $\left({ }^{*} p<0.05\right.$, Figure 14E).

\section{External Validations of the Six-Gene-Based Risk Score Using the GEO Database}

We downloaded GSE3218 and GSE10783 datasets from the GEO database to validate the risk assessment formula. However, the 

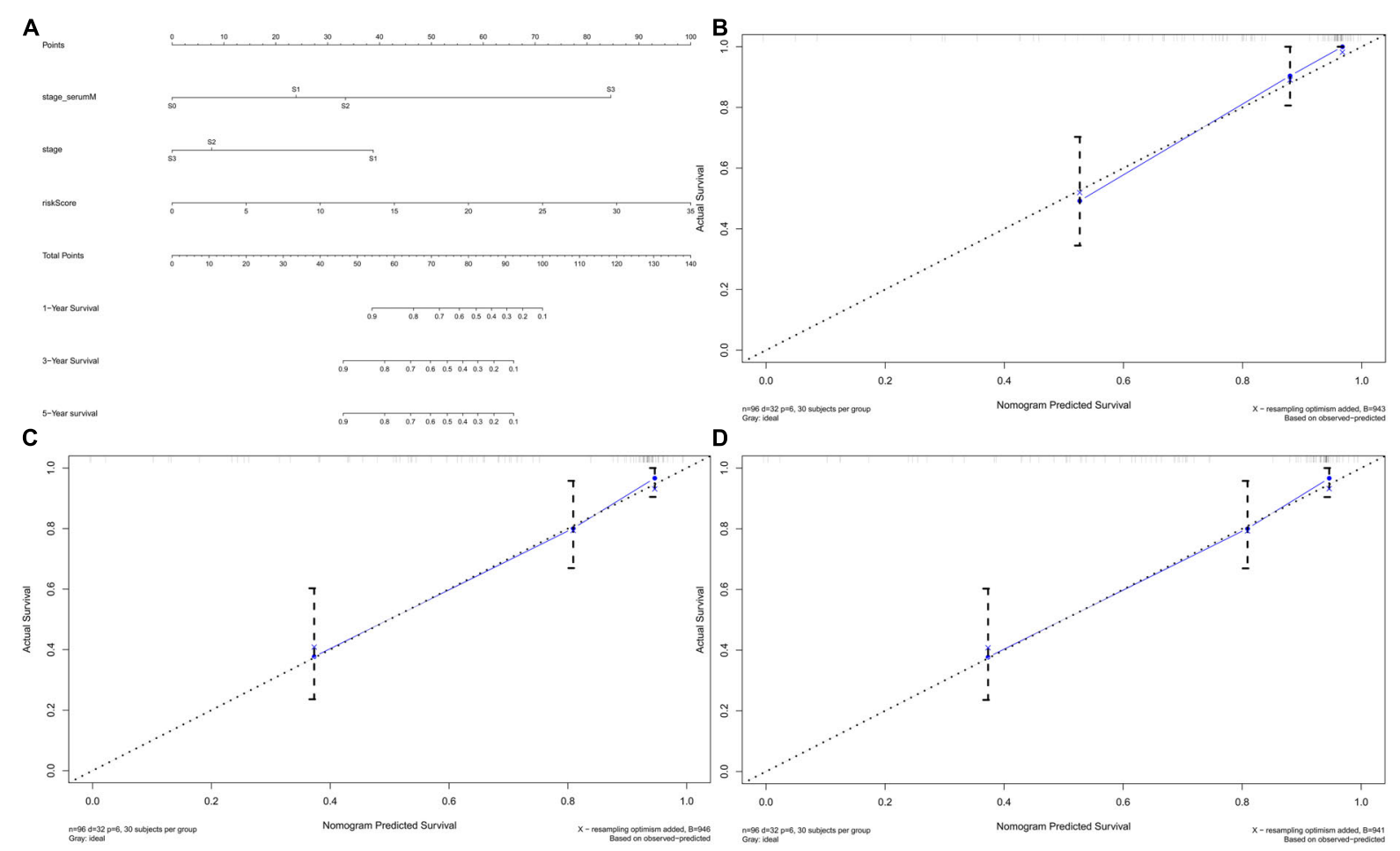

FIGURE 12 | Nomograms predicting survival probability of TGCT patients in TCGA. (A) Nomogram to predict 1-, 3-, and 5-year DFS. (B-D) Calibration plots of 1-, 3-, and 5-year DFS for nomograms. TCGA, The Cancer Genome Atlas; DFS, disease-free survival; TGCT, Testicular germ cell tumors.

risk score applied was inconsistent with the prognostic models we constructed previously. Higher risk group showed a better overall survival than low risk group ( $p=0.00001499$, Figure 15A). In terms of survival status the patients in high-risk cohorts have better OS than those in low-risk cohorts (Figures 15B,C). The reason for this adverse result is that the samples in GSE3218 and GSE10783 are mostly from patients with non-seminoma germ cell tumors (76 non-seminoma germ cell tumors, 15 embryonal carcinoma, 15 mature teratomas, 10 yolk sac tumors, 2 choriocarcinomas, 17 seminomas). In Figure 10, we found that this RBP-related risk score did not apply to patients with non-seminoma germ cell tumors. However, we cannot find other suitable GEO dataset for external validations.

\section{DISCUSSION}

In our current study, we screened six RBP genes with differential expression in patients with TGCT. Based on univariate and multivariate Cox analyses, we finally targeted six prognosisrelated RBPs to build the risk-scoring system. The prognosis prediction ability of the system underwent full verification in the test group, the entire TCGA cohort, and the GEO database. We observed that most of the numerical values of AUCs, which were calculated based on the risk scoring system in training group, test group, and entire TCGA cohort, were between 0.7 and 0.9, indicating that the systems showed fair or good performance to predict the DFS of patients with TGCT according to the traditional academic point system for classifying the accuracy of a diagnostic test. ${ }^{6}$ We also discovered that risk-scoring system and serum marker levels are important independent predictors of prognosis for patients with TGCT. The constructed nomogram was found reliable for clinical application.

After integrating information from the risk-score system with clinical characteristics, we found that the model performed well in predicting patients with different clinical characteristics except for patients with non-seminoma and M1 stage. These parameters represent early tumor stage and better survival outcome, which means that model could function well in predicting the survival of patients with relatively better conditions. We noticed that patients with TGCT have relatively better outcomes compared with patients with other malignant neoplasms, and more than 95\% of patients diagnosed with early stage TGCT can be cured (Lakomý et al., 2009). Researchers found that more than $80 \%$ of patients with TGCTs undergo conventional cisplatin-based chemotherapy could be cured, even at highly advanced stage, and thus exhibited good prognosis in TGCT (Einhorn, 2002).

The serum markers of TGCT, including AFP, hCG, and LDH, play crucial roles compared with other solid organ malignancies. A stage categorization of serum tumor markers stage category was created according to the levels of these serum markers. Markers with higher expression levels could predict worse TGCT outcome (Barlow et al., 2010). Based on our model, DFS decreased as risk scores rose in patients with TCGT who had

\footnotetext{
${ }^{6}$ http://gim.unmc.edu/dxtests/roc3.htm
} 


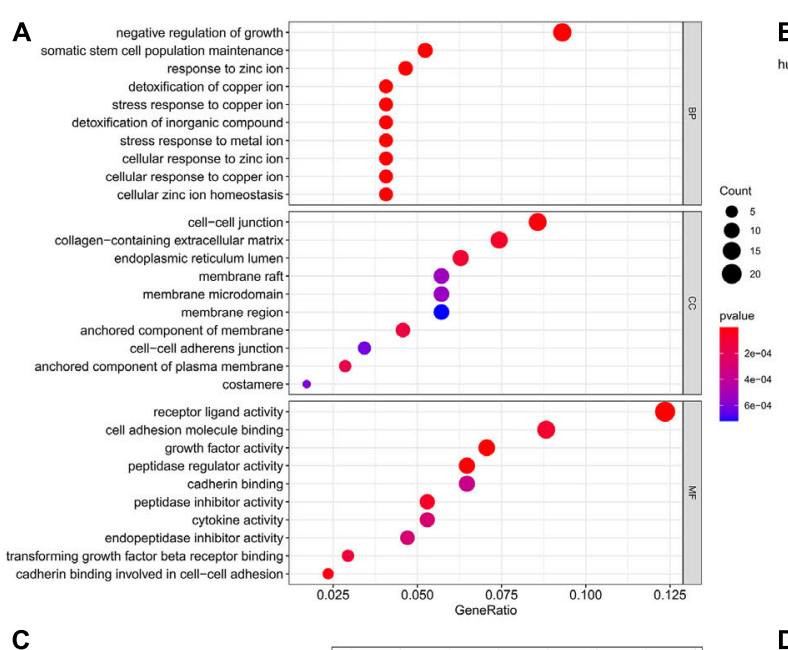

B complement activation, classical pathwa

rculating immunoglobuli

complement activation
protein activation cascade

immunoglobulin mediated immune respons
$\mathrm{B}$ cell mediated immunity

regulation of complement activation

gugution of protein activation cascade.
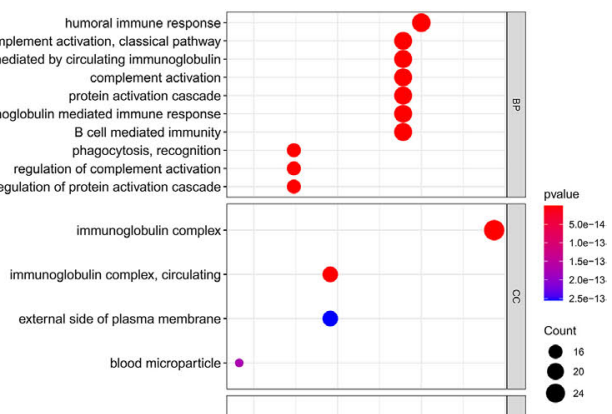

Signaling pathways regulating pluripotency of stem cell

Cytokine-cytokine receptor interaction

Mineral absorptio

Melanoma

Gastric cancer

Hippo signaling pathway

Proteoglycans in cancer

Biosynthesis of amino acids

TGF-beta signaling pathway

Breast cancer

HIIF-1 signaling pathw

Alanine, aspartate and glutamate metabolism

Cysteine and methionine metabolism.

Cortisol synthesis and secretion.

E
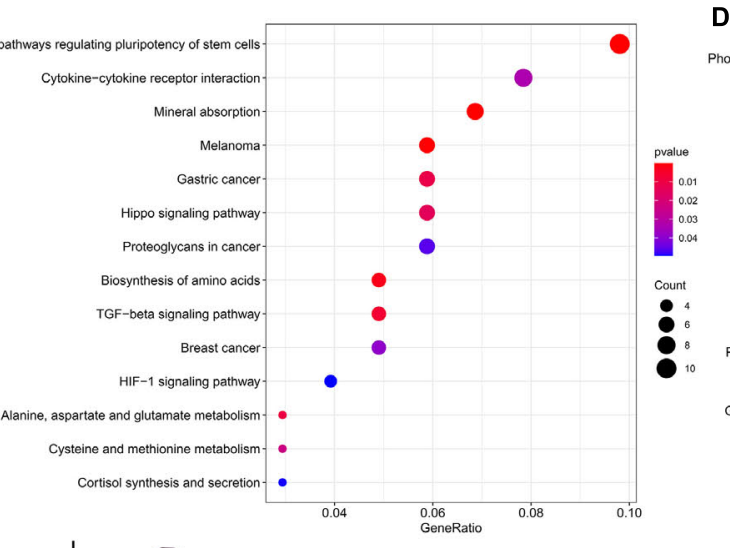

D
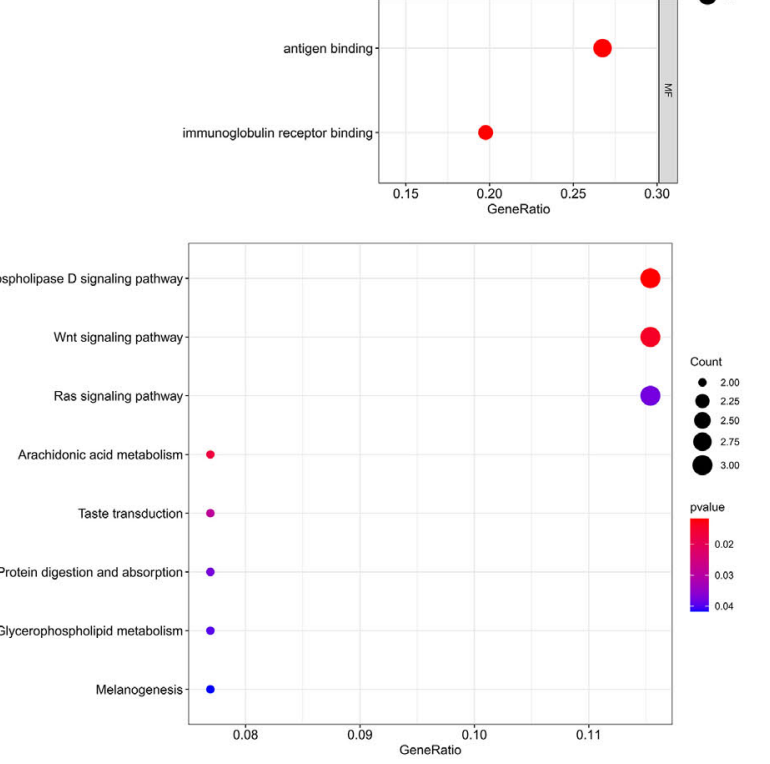
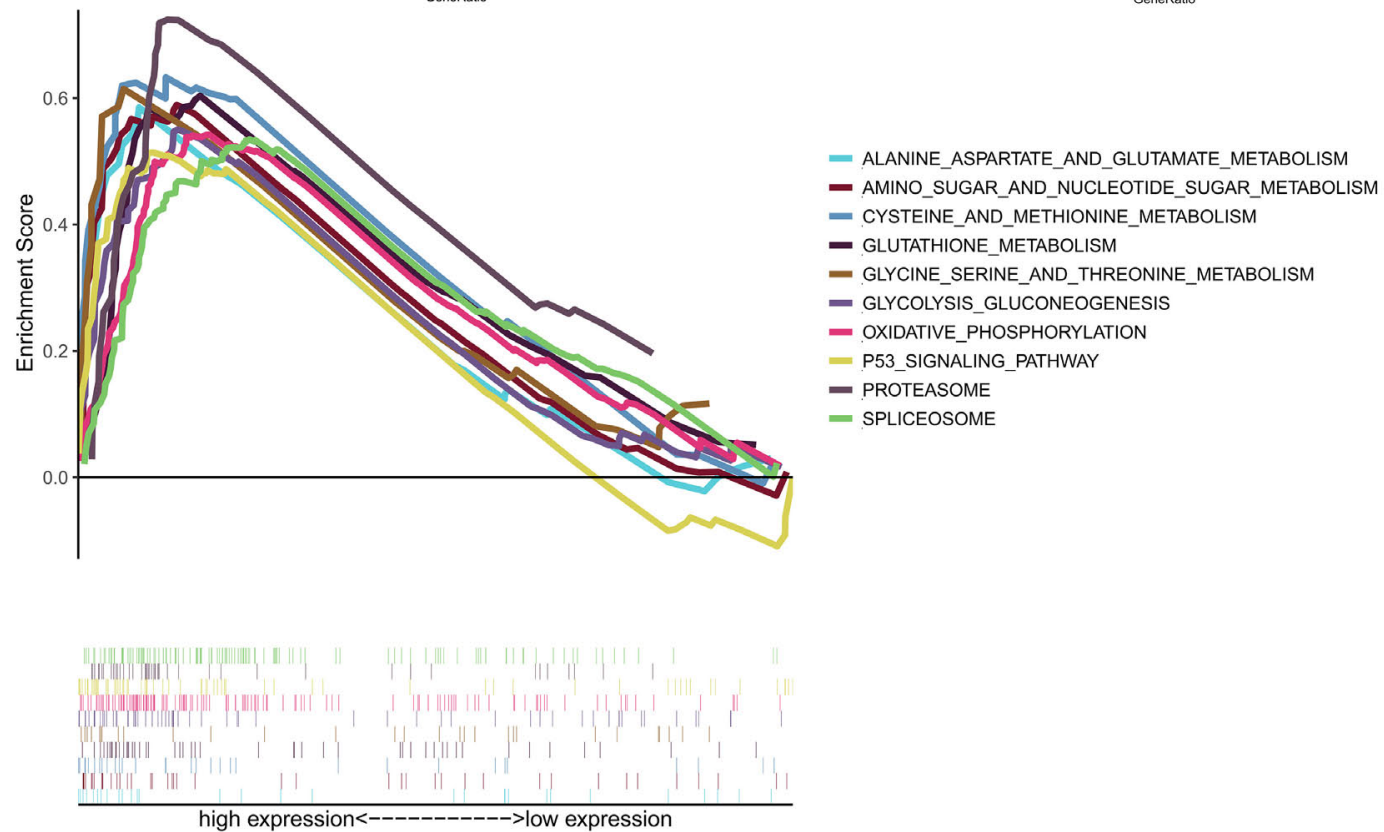

FIGURE 13 | Functional annotation of the differentially expressed genes between high risk and low risk groups based on six-RBP risk score of TGCT in TCGA cohort. Bubble plots showed enrichment of (A) GO terms associated with up-regulated genes and (B) GO terms associated with down-regulated genes. Bubble plots showed enrichment of (C) KEGG pathway associated with up-regulated genes and (D) KEGG pathway associated with down-regulated genes. (E) GSEA analysis showed the top ten most significantly enriched signaling pathways in high-risk score subgroup. GO, Gene Ontology; KEGG, Kyoto Encyclopedia of Genes and Genomes. 


\section{A}

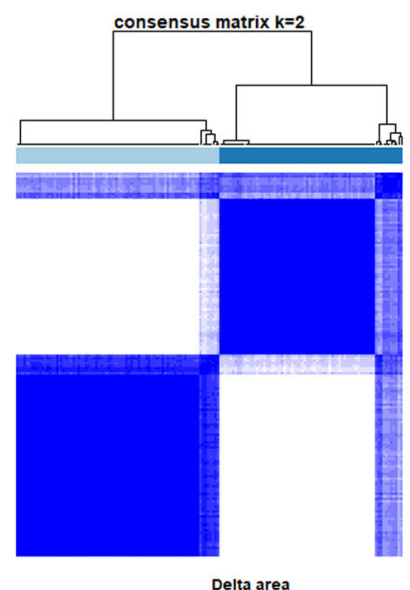

C

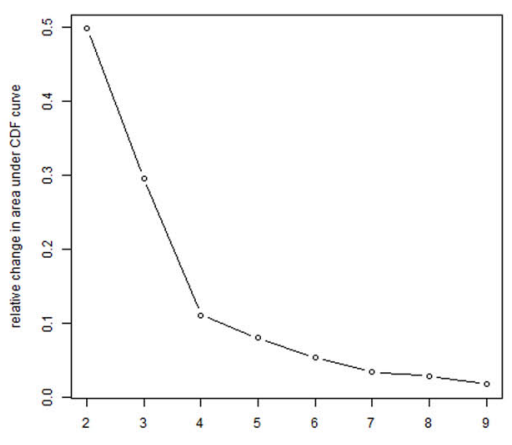

B
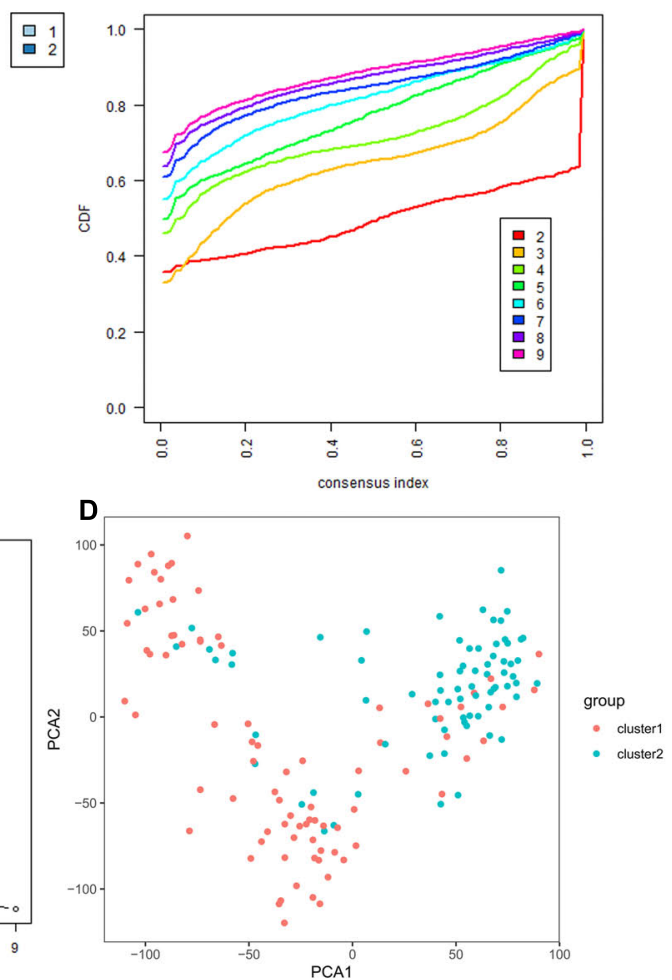

E

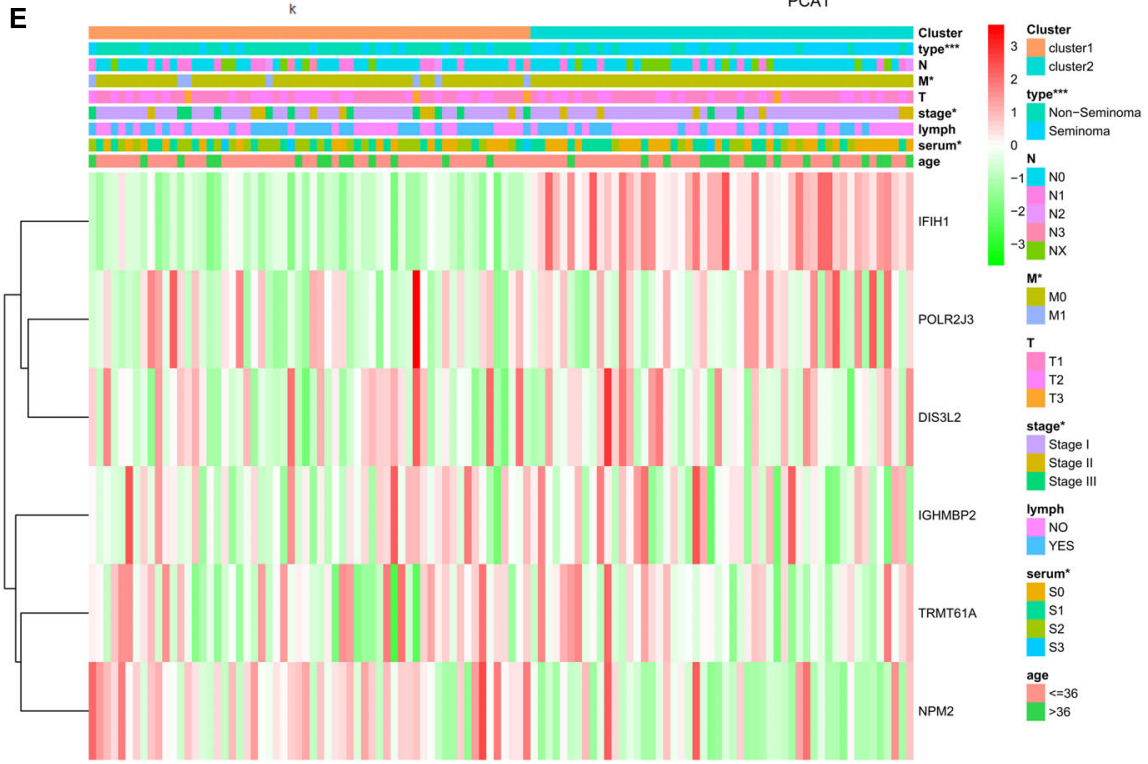

FIGURE 14 | Differential clinical characteristics of TGCT patients in the two different clusters. (A) Based on the expression similarity of RBPs, the TCGA TGCT cohort was separated into two distinct clusters when $k=2$. (B,C) Consensus CDF and relative change in area under CDF curve for $k=2-9$. (D) Principal component analysis based on two clusters. (E) Significant difference was observed for the type, grade, stage, and serum marker study levels between cluster 1 and cluster 2. CDF, clustering cumulative distribution function. ${ }^{*} p<0.05,{ }^{* *} p<0.01$, and ${ }^{* \star *} p<0.001$ compared with the cluster2 group. TGCT, Testicular germ cell tumors; RBPs, RNA-binding proteins; TCGA, The Cancer Genome Atlas.

serum marker levels within normal limits or beyond normal limits. We proposed an assumption that combination of the model and serum tumor markers may show better survival predicting function when the numerical values of traditional serum tumor markers of TCGT were within normal limits. AUCs of the risk score were all greater than 0.7, which implied its fair accuracy in prognosis prediction based on the above guidelines. Besides, the numerical values of AUCs were higher than other 
A

Risk \pm High risk \pm Low risk

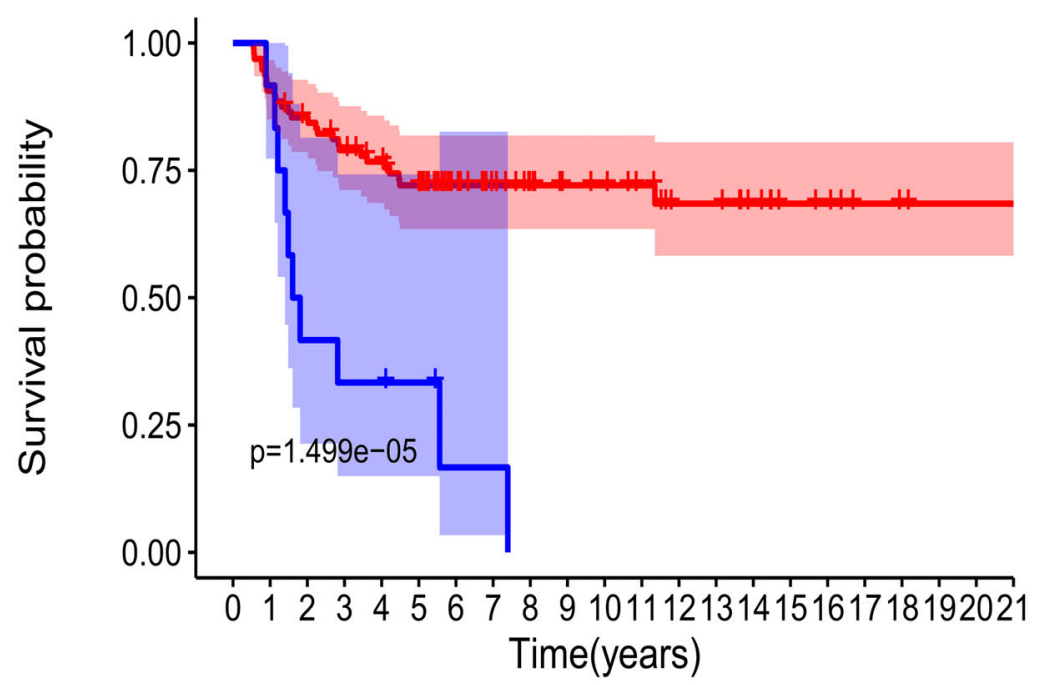

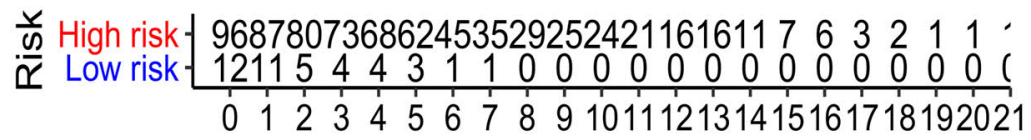

B

Time(years)
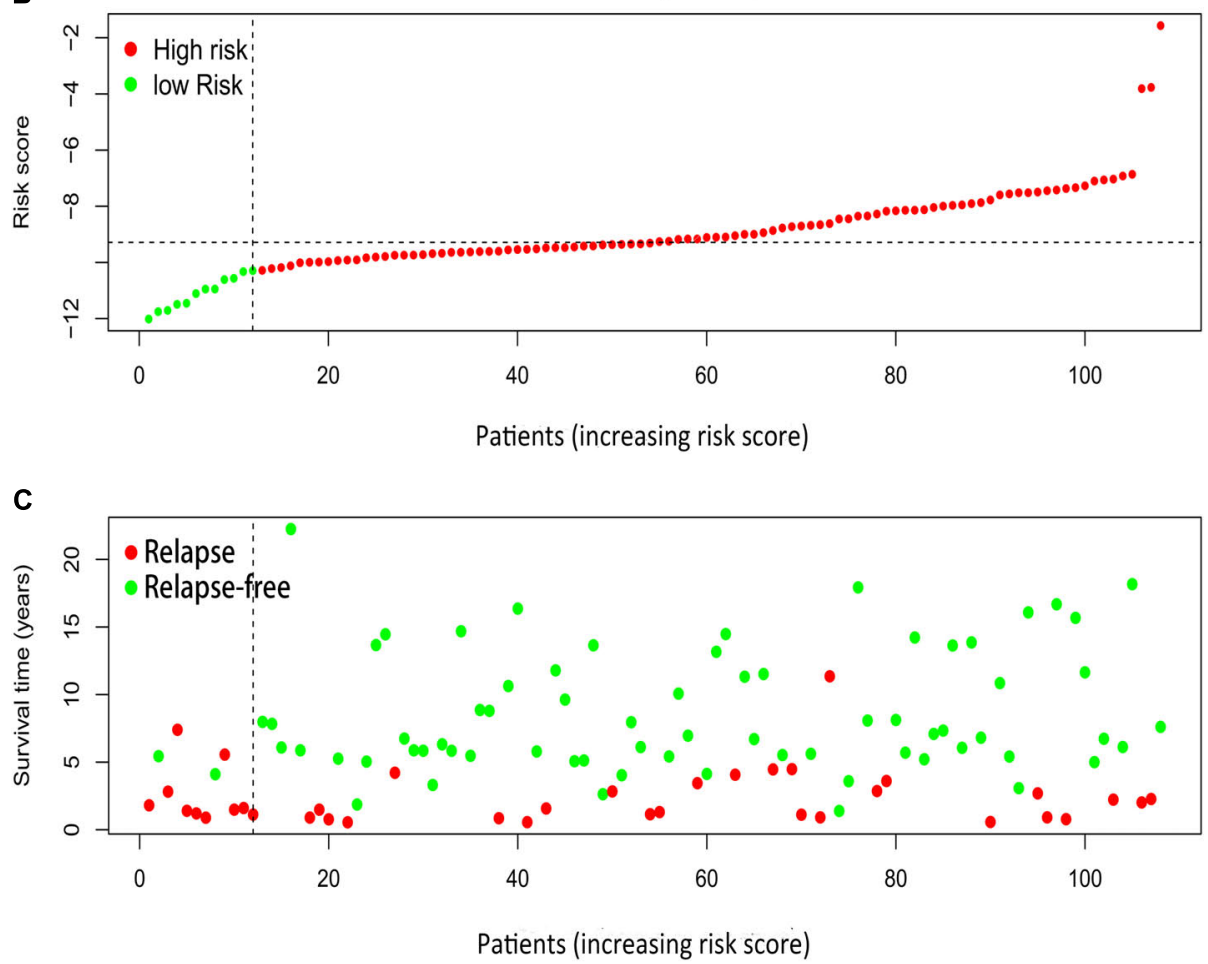

FIGURE 15 | Validation of the prognostic value of the risk model in GSE3218 and GSE10783 datasets. (A) Kaplan-Meier survival curve analysis of OS in high-risk score and low-risk score TGCT patients in GSE3218 and GSE10783 datasets, marked as red line and blue line separately. (B,C) Risk score, distribution of survival status between the high-risk and low-risk groups, and expression heat maps of six RBPs. OS, overall survival; TGCT, Testicular germ cell tumors; TCGA, The Cancer Genome Atlas; RBPs, RNA-binding proteins. 
factors, including serum marker levels, which suggested the model might serve as a better prognostic indicator.

We noticed that risk score seems higher in patients diagnosed with non-seminoma subtype or age $<36$ years old. This finding is reasonable because TGCTs are considered one of the most common malignancies in young adult males at the reproductive age (Hayes-Lattin and Nichols, 2009). However, Terbuch et al. found among metastatic TGCT cases that although higher age is associated with worse OS, it is not correlated with worse DFS or higher disease progression risk. Elderly patients could also gain high cure rates as younger patients if they tolerate riskadapted chemotherapy; thus, age might not remarkably affect the prognosis of metastatic TGCT cases (Terbuch et al., 2019).

Gene Ontology analysis results showed that BP terms concern chemical reactions on metallic ion. However, limited research looked into whether metallic ion plays a part in TGCT. Togawa et al. observed that paternal exposure to $\mathrm{Cr}$ might elevate the risk of TGCT in offspring. Although the evidence is limited (Togawa et al., 2016), more studies are needed to confirm whether environmental exposure to metal ion is a pathogenic factor of TGCT. KEGG analysis was conducted on basis of differentially expressed RBPs between "high-" and "low-risk score." "Signaling pathways regulating pluripotency of stem cells" was the most significantly enriched pathway. Differentiation arrest in male primordial germ cells during fetal development (the precursors of male germ line) is deemed as an origin of TGCT. SSC niche along with factors in the microenvironment of SSC might promote the development of some TGCT; hence, stem cells might play an important role in TGCT pathogenesis (Silván et al., 2013). Embryonal carcinomas (ECs), as a type of nonseminoma, often differentiates into teratoma, yolk sac tumor, and choriocarcinoma for its pluripotency (Dixon and Moore, 1953; Pierce and Abell, 1970). KLF4, overexpressed in seminoma cells, prevents differentiation and maintains proliferation and pluripotency during germ cell tumorigenesis; therefore, KLF4 may be vital in the neoplastic transformation of testicular stem cells. Similarly, stem cell factors, such as NANOG (Nettersheim et al., 2011) and OCT4 (Cheng et al., 2007), serve as markers in malignant human germ cell development. "Cytokine-cytokine receptor interaction pathways" were explored in previous studies in TGCT and ECs. CAR-T cells, considered effective as an immunotherapy for ECs, could eliminate EC cells via a cellcell contact-dependent Fas/FasL interaction. Fas/FasL interaction between tumor cells and CAR-T cells could be utilized for tumor escape reduction via elevating heterogeneous antigen expression or improving CAR T-cell antitumor activity (Hong et al., 2018). Guido et al. found that estradiol could induce PTEN gene expression by binding to ER $\beta$. ER $\beta / P T E N$ signaling induces cell death in seminoma cell lines by autophagy and necroptosis (Guido et al., 2012). Notably, the interaction between cytokines and their receptors were often discussed and proposed as therapeutic sites in the treatment of TGCT. The emergence of familial aggregations of TGCT has been described and suggests that hereditary susceptibility exists in the TGCT subset (Greene et al., 2010). In this study, we found "melanoma," "gastric cancer," and "breast cancer" pathways were also enriched in KEGG. This finding suggests that the molecular mechanisms of TGCT are partly consistent with other malignant tumors, which tend to have hereditary pathogenic factors as well. For instance, the lower expression of autophagy related proteins and the downregulation of autophagy lead to the acceleration of cancer cell proliferation in TGCT and melanoma (Liu et al., 2018). MiR-223-3p exerts oncogenic influence on TGCT by promoting cell proliferation and inhibiting apoptosis via FBXW7, which is regarded as a tumor suppresser. MiR-223$3 \mathrm{p}$ and FBXW7 also act as a target in gastric cancer (Liu et al., 2017). Overexpressed PTTG1 could cause aneuploidy and promotes oncogenesis in breast cancer (Watkins et al., 2010). Meanwhile, PTTG1 overexpression makes seminoma stem cells more invasive and aggravates neoplastic angiogenesis (Grande et al., 2019). The identification of targets in TGCT research can refer to mechanism research in other malignant tumors that possess hereditary susceptibility.

The GSEA results revealed that many pathways correlated with metabolism process, such as amnio acid metabolism (alanine, aspartate, and so on), sugar metabolism, and oxidative phosphorylation process, were enriched as risk score increased. This result was in cooperation with the KEGG results. Studies confirmed that metabolism is involved in the pathogenesis of TGCT. N-acetylcystein has negative impacts on bleomycininduced apoptosis in malignant TGCTs by inhibiting the mitochondrial pathway, which leads to resistance to apoptosis and might aggravate tumor progression (Cort et al., 2012). Four genes related to glucose metabolism (LDHA, MCT1, $P G K 1$, and TIGAR) exhibited remarkably high expression in TGCTs, and could be suppressed by microRNA-199a-3p, which might affect aerobic glycolysis and tumorigenesis, via binding to SP1 (Liu et al., 2016). Alterations in the expression level of proteins related to the Warburg effect in adult patients with TGCTs, such as GLUT1 and CD44, suggest the metabolic transformation of malignant cells toward a hyperglycolytic and acid-resistant phenotype in TGCTs, which have a worse outcome (Bonatelli et al., 2019). Researchers found that compared with embryonal carcinoma, the citric acid cycle/mitochondrial oxidative phosphorylation and sphingolipid biosynthesis in TGCT are decreased whereas arachidonic acid metabolism and long-chain fatty acid abundance are increased (Batool et al., 2019). In a word, metabolic reprogramming is common in TGCTs, and cancer therapy that targets metabolic process has brilliant prospects in the future.

P53 pathway, as a classic tumor-associated pathway, was enriched in the high-risk groups. The hyperactivation of p53 effectively enhances the susceptibility of TGCTs to chemotherapy (Kerley-Hamilton et al., 2005). TGCTs showed hypersensitivity to cisplatin because of the apoptosis induction effect of p53 (Gutekunst et al., 2011). The results of these studies imply that p53 activation has a protective effect and influence the effect of cancer treatment.

Some of the six prognostic RBPs are involved in cancer pathogenesis. DIS3L2, a conserved exoribonuclease, that plays a part in the degradation of cytoplasmic RNAs, is associated with several cancers. DIS3L2 promotes hepatocellular carcinoma progression via the regulation of hnRNP U-mediated alterative splicing (Xing et al., 2019). Partial or complete DIS3L2 
deficiency might cause an increased incidence of sporadic Wilms' tumor (Astuti et al., 2012). IFIH1, which acts as a cytosolic receptor, is essential for defense against viral infection by sensing double-stranded RNA (Fischer et al., 2020). IFIH1 is overexpressed in several topotecan-resistant ovarian cancer cell lines; thus, the gene might participate in the drug resistance mechanism of the tumor (Klejewski et al., 2017). IGHMBP2 encodes a helicase related to DNA replication and repair (Shen et al., 2006). IGHMBP2 promotes cell migration and invasion in esophageal squamous carcinoma by downregulating E-cadherin (Chunli et al., 2015). NPM2 is an oocyte-specific nuclear protein indispensable for nuclear and nucleolar organization. It also participates in early embryonic development processes (Lingenfelter et al., 2011). NPM2 is hypermethylated and downregulated in melanomas, thus, it might be involved in the early events in the development of malignant melanoma (Fujiwara et al., 2018). NPM2 is also regarded as a potential immunohistochemical marker via making a distinction between melanoma and benign 5 melanocytic lesions. In a word, these RBPs mentioned are correlated with the development of malignant tumors. POLR2J3 and TRMT61A are rarely found in malignancy before. The chosen RBPs might play a role in the pathogenesis and progression of TGCT, which requires further experiments in the future.

There are several potential reasons why the RBP-based model identified here may be superior to existing prognostic factors in determining the DFS risk for TGCT patients. First of all, the model was based on direct analysis of RBPs, which provides comprehensive and more precise information about the cellular events compared with either clinical parameters or hematological indexes. Second, the data is based on RNA sequencing, which is now a relatively affordable method to obtain an accurate picture of the abundance and activation state of RBPs. Third, the integration of RBPs with other prognostic factors in the nomogram model increased its reliability. Finally, compared with the widely used IGCCCG system, the prognostic predictive model described here contains more valuable information and is more user friendly, suggesting that it may have utility in clinical practice in the future. Based on this risk score, we can predict the DFS of TGCT patients and selected more aggressive therapy for patients with highrisk.

Limitations also exist in our research. First, the predictive efficiency of risk score is unsatisfactory for patients with nonseminoma. Furthermore, we found that this RBP-related risk score applied to seminoma germ cell tumors and did not apply to patients with non-seminoma germ cell tumors. Besides, further study is needed for the specific mechanism of these six RBPs.

\section{REFERENCES}

Astuti, D., Morris, M. R., Cooper, W. N., Staals, R. H., Wake, N. C., Fews, G. A., et al. (2012). Germline mutations in DIS3L2 cause the Perlman syndrome of overgrowth and Wilms tumor susceptibility. Nat. Genet. 44, 277-284. doi: 10.1038/ng.1071

Barlow, L. J., Badalato, G. M., and McKiernan, J. M. (2010). Serum tumor markers in the evaluation of male germ cell

\section{CONCLUSION}

Our results demonstrated that risk score based on six RBPs is associated with poor prognosis in patients with TGCT and can accurately predict prognosis. Risk score along with the serum markers of TGCT could serve as an independent risk factor of TGCT with clinical application in the future.

\section{DATA AVAILABILITY STATEMENT}

Publicly available datasets were analyzed in this study. This data can be found here: The Cancer Genome Atlas (https:// portal.gdc.cancer.gov/); the NCBI Gene Expression Omnibus (GSE3218 and GSE10783).

\section{AUTHOR CONTRIBUTIONS}

NS and XM: protocol/project development. LY and CJ: data collection or management. JL and XZ: data analysis. LY and RC: manuscript writing/editing. All authors read and approved the final manuscript.

\section{FUNDING}

This work was funded by the National Natural Science Foundation of China (Grant numbers: 81871151 and 81801438) and Medical key talent of Jiangsu Province: ZDRCA2016009.

\section{ACKNOWLEDGMENTS}

We would like to thank the researchers and study participants for their contributions.

\section{SUPPLEMENTARY MATERIAL}

The Supplementary Material for this article can be found online at: https://www.frontiersin.org/articles/10.3389/fgene. 2021.610291/full\#supplementary-material

Supplementary Figure 1 | The time-dependent ROC curves for six genes-based risk score, TRMT61A, POLR2J, DIS3L2, IFIH1, IGHMBP2, and NPM2 combining with 1- (A), 3- (B), 5- (C), and 10- (D) year DFS in TCGA TGCT cohort, respectively. DFS, disease-free survival. ROC, Receiver operating characteristic curve. TCGA, The Cancer Genome Atlas. TCGA, The Cancer Genome Atlas. DFS, disease-free survival. ROC, Receiver operating characteristic curve. TGCT, Testicular germ cell tumors.

tumors. Nat. Rev. Urol. 7, 610-617. doi: 10.1038/nrurol.20 10.166

Batool, A., Chen, S. R., and Liu, Y. X. (2019). Distinct metabolic features of seminoma and embryonal carcinoma revealed by combined transcriptome and metabolome analyses. J. Proteome Res. 18, 1819-1826. doi: 10.1021/acs. jproteome.9b00007

Bonatelli, M., Silva, E. C. A., Cárcano, F. M., Zaia, M. G., Lopes, L. F., Scapulatempo-Neto, C., et al. (2019). The warburg effect is associated with 
tumor aggressiveness in testicular germ cell tumors. Front. Endocrinol. 10:417. doi: 10.3389/fendo.2019.00417

Cheng, C. J., Wu, Y. C., Shu, J. A., Ling, T. Y., Kuo, H. C., Wu, J. Y., et al. (2007). Aberrant expression and distribution of the OCT-4 transcription factor in seminomas. J. Biomed. sci. 14, 797-807. doi: 10.1007/s11373-007-9198-7

Cheng, L., Albers, P., Berney, D. M., Feldman, D., Daugaard, G., Gilligan, T., et al. (2018). Testicular cancer. Nat. Rev. Dis. Primers 4:29.

Chovanec, M., Cierna, Z., Miskovska, V., Machalekova, K., Kalavska, K., Rejlekova, K., et al. (2018). $\beta$ catenin is a marker of poor clinical characteristics and suppressed immune infiltration in testicular germ cell tumors. BMC Cancer 18:1062. doi: 10.1186/s12885-018-4929-x

Chunli, W., Jiajie, H., Lifei, W., Beiqing, P., Xin, X., Yan, C., et al. (2015). [IGHMBP2 overexpression promotes cell migration and invasion in esophageal squamous carcinoma]. Yi chuan 37, 360-366.

Cort, A., Timur, M., Dursun, E., Kucuksayan, E., Aslan, M., and Ozben, T. (2012). Effects of $\mathrm{N}$-acetylcystein on bleomycin-induced apoptosis in malignant testicular germ cell tumors. J. Physiol. Biochem. 68, 555-562. doi: 10.1007/ s13105-012-0173-z

Diamantopoulos, N., and Kortsaris, A. (2010). Testicular germ cell tumors. J. BUON 15, 421-434.

Dixon, F. J., and Moore, R. A. (1953). Testicular tumors; a clinicopathological study. Cancer 6, 427-454.

Einhorn, L. H. (2002). Chemotherapeutic and surgical strategies for germ cell tumors. Chest Surg. Clin. N. Am. 12, 695-706. doi: 10.1016/s1052-3359(02) 00029-7

Fischer, H., Tschachler, E., and Eckhart, L. (2020). Pangolins lack IFIH1/MDA5, a cytoplasmic RNA sensor that initiates innate immune defense upon coronavirus infection. Front. Immunol. 11:939. doi: 10.3389/fimmu.2020.00939

Fujiwara, S., Nagai, H., Jimbo, H., Jimbo, N., Tanaka, T., Inoie, M., et al. (2018). Gene expression and methylation analysis in melanomas and melanocytes from the same patient: loss of NPM2 expression is a potential immunohistochemical marker for melanoma. Front. Oncol. 8:675. doi: 10.3389/fonc.2018.00675

Gerstberger, S., Hafner, M., and Tuschl, T. (2014). A census of human RNA-binding proteins. Nat. Rev. Genet. 15, 829-845. doi: 10.1038/nrg3813

Glisovic, T., Bachorik, J. L., Yong, J., and Dreyfuss, G. (2008). RNA-binding proteins and post-transcriptional gene regulation. FEBS Lett. 582, 1977-1986. doi: 10.1016/j.febslet.2008.03.004

Grande, G., Milardi, D., Martini, M., Cenci, T., Gulino, G., Mancini, F., et al. (2019). Protein expression of PTTG-1, OCT-4, and KLF-4 in seminoma: a pilot study. Front. Endocrinol. 10:619. doi: 10.3389/fendo.2019.00619

Greene, M. H., Kratz, C. P., Mai, P. L., Mueller, C., Peters, J. A., Bratslavsky, G., et al. (2010). Familial testicular germ cell tumors in adults: 2010 summary of genetic risk factors and clinical phenotype. Endocr. Relat. Cancer 17, R109-R121.

Guido, C., Panza, S., Santoro, M., Avena, P., Panno, M. L., Perrotta, I., et al. (2012). Estrogen receptor beta $(\mathrm{ER} \beta)$ produces autophagy and necroptosis in human seminoma cell line through the binding of the Sp1 on the phosphatase and tensin homolog deleted from chromosome 10 (PTEN) promoter gene. Cell Cycle (Georgetown Tex) 11, 2911-2921. doi: 10.4161/cc.21336

Gutekunst, M., Oren, M., Weilbacher, A., Dengler, M. A., Markwardt, C., Thomale, J., et al. (2011). p53 hypersensitivity is the predominant mechanism of the unique responsiveness of testicular germ cell tumor (TGCT) cells to cisplatin. PLoS One 6:e19198. doi: 10.1371/journal.pone.0019198

Hayes-Lattin, B., and Nichols, C. R. (2009). Testicular cancer: a prototypic tumor of young adults. Semin. Oncol. 36, 432-438. doi: 10.1053/j.seminoncol.2009. 07.006

Hong, L. K., Chen, Y., Smith, C. C., Montgomery, S. A., Vincent, B. G., Dotti, G., et al. (2018). CD30-redirected chimeric antigen receptor T cells target $\mathrm{CD} 30(+)$ and $\mathrm{CD} 30(-)$ embryonal carcinoma via antigen-dependent and Fas/FasL interactions. Cancer Immunol. Res. 6, 1274-1287. doi: 10.1158/23266066.cir-18-0065

Kerley-Hamilton, J. S., Pike, A. M., Li, N., DiRenzo, J., and Spinella, M. J. (2005). A p53-dominant transcriptional response to cisplatin in testicular germ cell tumor-derived human embryonal carcinoma. Oncogene 24, 6090-6100. doi: 10.1038/sj.onc. 1208755

Klejewski, A., Świerczewska, M., Zaorska, K., Brązert, M., Nowicki, M., Zabel, M., et al. (2017). New and old genes associated with topotecan resistance development in ovarian cancer cell lines. Anticancer Res. 37, 1625-1636. doi: 10.21873/anticanres. 11493
Lakomý, R., Poprach, A., Nēmecek, R., Vyskocil, J., Ondrová, B., Kocák, I., et al. (2009). [Adjuvant treatment for stage I of testicular germ cell tumours]. Klin. Onkol. 22, 22-26.

Li, H., Liang, Z., Yang, J., Wang, D., Wang, H., Zhu, M., et al. (2019). DAZL is a master translational regulator of murine spermatogenesis. Natl. Sci. Rev. 6, 455-468. doi: 10.1093/nsr/nwy163

Li, L. J., Zhang, F. B., Liu, S. Y., Tian, Y. H., Le, F., Lou, H. Y., et al. (2014). Decreased expression of SAM68 in human testes with spermatogenic defects. Fertil. Steril. 102, 61-67.e3.

Lingenfelter, B. M., Tripurani, S. K., Tejomurtula, J., Smith, G. W., and Yao, J. (2011). Molecular cloning and expression of bovine nucleoplasmin 2 (NPM2): a maternal effect gene regulated by miR-181a. Reprod. Biol. Endocrinol. 9:40. doi: 10.1186/1477-7827-9-40

Liu, H., He, Z., Bode, P., Moch, H., and Simon, H. U. (2018). Downregulation of autophagy-related proteins 1,5 , and 16 in testicular germ cell tumors parallels lowered LC3B and elevated p62 levels, suggesting reduced basal autophagy. Front. Oncol. 8:366. doi: 10.3389/fonc.2018.00366

Liu, J., Shi, H., Li, X., Chen, G., Larsson, C., and Lui, W. O. (2017). miR-223$3 \mathrm{p}$ regulates cell growth and apoptosis via FBXW7 suggesting an oncogenic role in human testicular germ cell tumors. Int. J. Oncol. 50, 356-364. doi: 10.3892/ijo.2016.3807

Liu, X., Duan, H., Zhou, S., Liu, Z., Wu, D., Zhao, T., et al. (2016). microRNA-199a$3 \mathrm{p}$ functions as tumor suppressor by regulating glucose metabolism in testicular germ cell tumors. Mol. Med. Rep. 14, 2311-2320. doi: 10.3892/mmr.2016.5472

Lü, M., Tian, H., Cao, Y. X., He, X., Chen, L., Song, X., et al. (2015). Downregulation of miR-320a/383-sponge-like long non-coding RNA NLC1-C (narcolepsy candidate-region 1 genes) is associated with male infertility and promotes testicular embryonal carcinoma cell proliferation. Cell Death Dis. 6:e1960. doi: 10.1038/cddis.2015.267

Marshall, C., Enzerra, M., Rahnemai-Azar, A. A., and Ramaiya, N. H. (2019). Serum tumor markers and testicular germ cell tumors: a primer for radiologists. Abdom. Radiol. (N. Y.) 44, 1083-1090. doi: 10.1007/s00261-018-1846-Z

Martinelli, C., Lengert, A. V. H., Cárcano, F. M., Silva, E. C. A., Brait, M., Lopes, L. F., et al. (2017). MGMT and CALCA promoter methylation are associated with poor prognosis in testicular germ cell tumor patients. Oncotarget 8, 50608-50617. doi: 10.18632/oncotarget.11167

Nettersheim, D., Biermann, K., Gillis, A. J., Steger, K., Looijenga, L. H., and Schorle, H. (2011). NANOG promoter methylation and expression correlation during normal and malignant human germ cell development. Epigenetics 6, 114-122. doi: 10.4161/epi.6.1.13433

Oechsle, K., Honecker, F., Cheng, T., Mayer, F., Czaykowski, P., Winquist, E., et al. (2011). Preclinical and clinical activity of sunitinib in patients with cisplatinrefractory or multiply relapsed germ cell tumors: a canadian urologic oncology group/german testicular cancer study group cooperative study. Ann. Oncol. 22, 2654-2660. doi: 10.1093/annonc/mdr026

Pereira, B., Billaud, M., and Almeida, R. (2017). RNA-binding proteins in cancer: old players and new actors. Trends Cancer 3, 506-528. doi: 10.1016/j.trecan. 2017.05.003

Pierce, G. B., and Abell, M. R. (1970). Embryonal carcinoma of the testis. Pathol. Annu. 5, 27-60.

Shen, J., Terry, M. B., Gammon, M. D., Gaudet, M. M., Teitelbaum, S. L., Eng, S. M., et al. (2006). IGHMBP2 Thr671Ala polymorphism might be a modifier for the effects of cigarette smoking and PAH-DNA adducts to breast cancer risk. Breast Cancer Res. Treat. 99, 1-7. doi: 10.1007/s10549-006-9174-3

Silván, U., Díez-Torre, A., Moreno, P., Arluzea, J., Andrade, R., Silió, M., et al. (2013). The spermatogonial stem cell niche in testicular germ cell tumors. Int. J. Dev. Biol. 57, 185-195. doi: 10.1387/ijdb.130068ja

Subramanian, A., Tamayo, P., Mootha, V. K., Mukherjee, S., Ebert, B. L., Gillette, M. A., et al. (2005). Gene set enrichment analysis: a knowledge-based approach for interpreting genome-wide expression profiles. Proc. Natl. Acad. Sci. U.S.A. 102, 15545-15550. doi: 10.1073/pnas.0506580102

Terbuch, A., Posch, F., Bauernhofer, T., Pichler, M., Peinsith, H., Szkandera, J., et al. (2019). Age as a predictor of treatment outcome in metastatic testicular germ cell tumors. Anticancer Res. 39, 5589-5596. doi: 10.21873/anticanres. 13753

Togawa, K., Le Cornet, C., Feychting, M., Tynes, T., Pukkala, E., Hansen, J., et al. (2016). Parental occupational exposure to heavy metals and welding fumes and risk of testicular germ cell tumors in offspring: a registry-based case-control 
study. Cancer Epidemiol. Biomarkers Prev. 25, 1426-1434. doi: 10.1158/10559965.epi-16-0328

Tsili, A. C., Sofikitis, N., Stiliara, E., and Argyropoulou, M. I. (2019). MRI of testicular malignancies. Abdom. Radiol. (N. Y.) 44, 1070-1082. doi: 10.1007/ s00261-018-1816-5

Watkins, R. J., Read, M. L., Smith, V. E., Sharma, N., Reynolds, G. M., Buckley, L., et al. (2010). Pituitary tumor transforming gene binding factor: a new gene in breast cancer. Cancer Res. 70, 3739-3749. doi: 10.1158/0008-5472.can09-3531

Xing, S., Li, Z., Ma, W., He, X., Shen, S., Wei, H., et al. (2019). DIS3L2 promotes progression of hepatocellular carcinoma via hnRNP U-mediated alternative splicing. Cancer Res. 79, 4923-4936. doi: 10.1158/0008-5472.can-190376

Yu, J., Navickas, A., Asgharian, H., Culbertson, B., Fish, L., Garcia, K., et al. (2020). RBMS1 suppresses colon cancer metastasis through targeted stabilization of its mRNA regulon. Cancer Discov. 10, 1410-1423. doi: 10.1158/2159-8290.cd-191375
Zhang, T., Ji, L., Liu, B., Guan, W., Liu, Q., and Gao, Y. (2018). Testicular germ cell tumors: a clinicopathological and immunohistochemical analysis of 145 cases. Int. J. Clin. Exp. Pathol. 11, 4622-4629.

Zhao, J., Zhang, Y., Liu, X. S., Zhu, F. M., Xie, F., Jiang, C. Y., et al. (2020). RNAbinding protein Musashi2 stabilizing androgen receptor drives prostate cancer progression. Cancer Sci. 111, 369-382. doi: 10.1111/cas.14280

Conflict of Interest: The authors declare that the research was conducted in the absence of any commercial or financial relationships that could be construed as a potential conflict of interest.

Copyright (c) 2021 Yao, Cong, Ji, Zhou, Luan, Meng and Song. This is an open-access article distributed under the terms of the Creative Commons Attribution License (CC BY). The use, distribution or reproduction in other forums is permitted, provided the original author(s) and the copyright owner(s) are credited and that the original publication in this journal is cited, in accordance with accepted academic practice. No use, distribution or reproduction is permitted which does not comply with these terms. 